\title{
Time Consistency Conditions for Acceptability Measures, with an Application to Tail Value at Risk
}

\author{
Berend Roorda* $\quad$ Hans Schumacher ${ }^{\dagger}$
}

April 12, 2005

\begin{abstract}
An acceptability measure is a number that summarizes information on monetary outcomes of a given position in various scenarios, and that, depending on context, may be interpreted as a capital requirement or as a price. In a multiperiod setting, it is reasonable to require that an acceptability measure should satisfy certain conditions of time consistency. Various notions of time consistency may be considered. Within the framework of coherent risk measures as proposed by Artzner et al. (1999), we establish implication relations between a number of different notions, and we determine how each notion of time consistency is expressed through properties of a representing set of test measures. We propose modifications of the standard Tail-Value-at-Risk measure that have stronger consistency properties than the original.

Keywords: coherent risk measures; acceptability measures; nonlinear expectations; capital requirements; incomplete markets; time consistency.
\end{abstract}

\section{Introduction}

Consider a position ${ }^{1}$ giving rise to monetary outcomes that may be different in different scenarios. After a set of scenarios has been constructed and outcomes in each scenario

${ }^{*}$ B. Roorda, FELab and Department of Technology and Management, University of Twente, P.O. Box 217, 7500 AE, Enschede, the Netherlands. Phone: +3153-4894383. E-mail: b.roorda@utwente.nl. Part of this research was supported by the Netherlands Organization for Scientific Research (NWO - MaGW/ESR, project number 510-01-0025).

${ }^{\dagger}$ J.M. Schumacher, CentER and Department of Econometrics and Operations Research, Tilburg University, P.O. Box 90153, 5000 LE Tilburg, the Netherlands.

${ }^{1}$ We use the term "position" here to refer to any project or contract subject to evaluation on the basis of positive or negative future payoffs. A position may for instance consist of a derivative contract, a portfolio of insurance policies, or the collection of assets and liabilities of a pension fund. 
have been determined, one frequently needs to summarize all of this information in a single number (an "acceptability measure") which, depending on the particular application, may for instance be interpreted as a capital requirement or as a price. To determine which acceptability measure is appropriate for a given situation, one may make use of axiom systems and representation theorems. The specification of axioms helps to clarify differences between various acceptability measures that may be proposed. Given a collection of axioms, it is sometimes possible to prove a representation theorem providing a concrete form for all measures that satisfy the given axioms.

The discussion of axiom systems for acceptability measures has received a new impulse after capital adequacy rules proposed in the Basel Accord and elsewhere were investigated from an axiomatic point of view by Artzner et al. (1999). The proposed set of axioms defining "coherent" acceptability measures ${ }^{2}$ is different from the sets of axioms traditionally used in statistical decision theory and admits a relatively simple representation theorem. Although the work of Artzner et al. (1999) is formulated in a supervisory framework, the principles of the approach may be applied as well to insurance premium determination and to asset pricing, so that there are connections to the extensive literature on premium principles and to the more recent work on "good-deal pricing" in incomplete markets. From a mathematical point of view, acceptability measures may be viewed as nonlinear expectations, which brings in a connection to yet another strand of research. For an entry to the related literature, see for instance Carr et al. (2001), Černý and Hodges (2002), Föllmer and Schied (2004), Frittelli and Rosazza Gianin (2002), Jaschke and Küchler (2001), Kaas et al. (2003), Landsman and Sherris (2001), Peng (1997), and Wang et al. (1997).

The work of Artzner et al. (1999) is formulated in a single-period setting. In many applications, though, it may be envisaged that acceptability measures for a given position will be computed at more than one moment in time, and that acceptability may be enhanced by the use of dynamic strategies. Considerable effort has recently been spent on the development of axiom systems and representation results that apply to dynamic settings (Artzner et al. 2004, Epstein and Schneider 2003, Frittelli and Scandolo 2004, Wang 2003, Riedel 2004, Roorda et al. 2004, Weber 2004). In the extension to the dynamic case, a key role is played by notions of time consistency. It is the purpose of the present paper to establish the relations between a number of different axiomatizations of the idea of time consistency, working in a finite context (for simplicity) and within the setting of coherent acceptability measures. Straightforward multiperiod extensions of single-period coherent acceptability

\footnotetext{
${ }^{2}$ The cited paper actually employs an opposite sign convention to the one used here and the term "risk measure" is used rather than "acceptability measure". In later work by Artzner and co-authors (Artzner et al. 2004), the same sign convention is used as we do in this paper, and the term "risk-adjusted value" is used for what we call "acceptability measure".
} 
measures may well give rise to consistency problems, as noted by Artzner (2002) (see also Artzner et al. (2004)) for the case of the Tail-Value-at-Risk measure. We discuss adaptations of this measure which enjoy better consistency properties.

The paper distinguishes essentially three different notions of time consistency, which under mild assumptions are ordered by implication relations. The weakest notion is called conditional consistency. This notion requires that the evaluation of a conditional version of a given payoff should be in line with the conditional evaluation of that payoff. The second notion we consider is called sequential consistency. This form of time consistency requires that a position cannot be evaluated positively if all conditional evaluations at later stages are negative. The third and strongest notion is called dynamic consistency. This notion has been used extensively in the recent work on dynamic risk measures. It is shown here to be closely related to what might be called the tower law of conditional evaluations, which holds for a given acceptability measure if evaluations under this measure do not change when the payoffs following a given future event are replaced by their evaluation conditional on that event.

The paper is organized as follows. In Section 2 we summarize the framework. Section 3 introduces the notions of time consistency that we discuss in this paper and that were already mentioned above. Characterizations of these concepts in terms of single-step properties are provided in Section 4. In Section 5 we establish implication relations between different notions of consistency, and we show by examples that the notions we introduce are indeed not equivalent. A major theme in the theory of coherent acceptability measures is representation by means of collections of probability measures. Various representation forms of this type for multiperiod acceptability measures are discussed in Section 6. Subsequently, in Section 7, relations are established between consistency notions and representation properties. Applications to TailVaR are discussed in Section 8, and conclusions follow in Section 9. Most of the proofs of the results of this paper have been collected in an Appendix.

Throughout the paper, we assume that payoffs are represented in terms of a suitable numéraire so that effectively we may suppose that interest rates are zero. This assumption is commonly made in the literature on dynamic acceptability measures; it simplifies exposition considerably without imposing any essential constraint. As a matter of notation: given a set $\mathcal{S} \subseteq \mathbb{R}^{n}$, its closure in the usual topology of $\mathbb{R}^{n}$ is denoted by cl $\mathcal{S}$ or $\overline{\mathcal{S}}$, its convex hull is written as $\operatorname{ch} \mathcal{S}$, and its closed convex hull is indicated by $\operatorname{cch} \mathcal{S}:=\operatorname{cl} \operatorname{ch} \mathcal{S}$. 


\section{Mathematical framework}

\subsection{Single-period acceptability measures}

Let $\Omega$ be a finite set, say with $n$ elements. The set of all functions from $\Omega$ to $\mathbb{R}$ will be denoted by $\mathcal{X}(\Omega)\left(\simeq \mathbb{R}^{n}\right)$. An element $X$ of $\mathcal{X}(\Omega)$ represents the position that generates outcome $X(\omega)$ when the state $\omega \in \Omega$ arises. A (single-period) acceptability measure defined on $\Omega$ is a mapping from $\mathcal{X}(\Omega)$ to $\mathbb{R}$. The number $\phi(X)$ that is associated to the position $X \in \mathcal{X}(\Omega)$ by an acceptability measure $\phi$ is interpreted as the "degree of acceptability" of the position $X$; if $\phi(X) \geq 0, X$ is called acceptable. An acceptability measure is said to be coherent (Artzner et al., 1999) if it satisfies the following four axioms, where $\mathbb{1}: \Omega \rightarrow \mathbb{R}$ is defined by $\mathbb{1}(\omega)=1$ for all $\omega$ :

- translation property: $\phi(X+\eta \mathbb{1})=\phi(X)+\eta$ for all $\eta \in \mathbb{R}$

- superadditivity: $\phi(X+Y) \geq \phi(X)+\phi(Y)$

- positive homogeneity: $\phi(\lambda X)=\lambda \phi(X)$ for all $\lambda \geq 0$

- monotonicity: $X \geq Y$ implies $\phi(X) \geq \phi(Y)$.

Here we write $X \geq Y$ if $X(\omega) \geq Y(\omega)$ for all $\omega \in \Omega$. Instead of $\eta \mathbb{1}$, we also often write simply $\eta$.

A general motivation for the above principles is provided in Artzner et al. (1999). The translation property, together with the positive homogeneity property applied with $\lambda=0$, implies in particular that $\phi(\eta)=\eta$ for all $\eta \in \mathbb{R}$. Consequently, we have $\phi(\phi(X))=$ $\phi(X)$ for all positions $X$ which means that $\phi(X)$ may be seen as a certainty equivalent of $X$. The positive homogeneity and superadditivity axioms may be relaxed to a convexity axiom (Föllmer and Schied 2002). We do not consider this generalization here. Positive homogeneity may be justified in cases where risks can be split between many parties if necessary.

The basic representation theorem of coherent acceptability measures can be stated as follows.

Theorem 2.1 (Artzner et al. (1999, Prop. 4.1); Huber (1981, Prop. 10.2.1)) An acceptability measure $\phi$ defined on a finite set $\Omega$ is coherent if and only if there exists a family $\mathcal{P}$ of probability measures on $\Omega$ such that, for all $X \in \mathcal{X}(\Omega)$,

$$
\phi(X)=\inf _{\mathbb{P} \in \mathcal{P}} E_{\mathbb{P}} X
$$


The acceptability measure associated to a test set $\mathcal{P}$ does not change when $\mathcal{P}$ is replaced by its closed ${ }^{3}$ convex hull cch $\mathcal{P}$. It follows from a standard separating hyperplane argument that the closed convex set of test measures representing a given coherent acceptability measure is determined uniquely.

\section{$2.2 \quad$ Multiperiod setting}

Throughout the paper, the following structure is assumed. Time is discrete and runs from 0 to $T$; the initial state at time 0 is fixed. The sample space (scenario set) $\Omega$ is the set of all sequences $\left(\alpha_{1}, \ldots, \alpha_{T}\right)$ with $\alpha_{i} \in A$, where $A$ is a given finite set of "events." The set of all probability measures on $\Omega$ is denoted by $\operatorname{Pr}$.

In this context, we use the following notation. The symbols $\Omega^{\prime}$ and $\Omega^{\prime \prime}$ denote the collections of all sequences $\left(\alpha_{1}, \ldots, \alpha_{t}\right)$ of length $t \leq T$ and of length $t<T$ respectively. The length of a sequence $\omega^{\prime} \in \Omega^{\prime}$ is denoted by $T\left(\omega^{\prime}\right)$. The set $\Omega_{0}$ of sequences of zero length consists of a single element that we denote by 0 . Elements of $\Omega^{\prime}$ are referred to as partial histories or (with the tree interpretation in mind) as nodes.

We say that $\omega^{\prime} \in \Omega^{\prime}$ precedes $\omega \in \Omega$ if there are $\alpha_{t+1}, \ldots, \alpha_{T} \in A$ such that $\omega=$ $\left(\omega^{\prime}, \alpha_{t+1}, \ldots, \alpha_{T}\right)$, and in this case we write $\omega \succeq \omega^{\prime}$. We also write $F\left(\omega^{\prime}\right):=\left\{\omega \in \Omega \mid \omega \succeq \omega^{\prime}\right\}$, and use $\mathcal{F}_{t}$ to denote the algebra generated by the sets $F\left(\omega^{\prime}\right)$ with $\omega^{\prime}$ in the set $\Omega_{t}$ of sequences of length exactly $t$. The restriction of $\omega$ to the time period of length $t$ is denoted by $\left.\omega\right|_{t}$. The notion of a stopping time is defined in the usual way: a stopping time $\tau$ is a mapping from $\Omega$ to $\{0, \ldots, T\}$ such that, for all $t \in\{0, \ldots, T\}$, we have $\{\omega \mid \tau(\omega) \leq t\} \in \mathcal{F}_{t}$. The notation $\left.\omega\right|_{t}$ can be extended in a natural way to stopping times, and $\Omega_{\tau}:=\left\{\left.\omega\right|_{\tau(\omega)}\right\}_{\omega \in \Omega}$ denotes the set of stopping nodes corresponding to $\tau$. The set of all probability measures on $F\left(\omega^{\prime}\right)$, for given $\omega^{\prime} \in \Omega^{\prime}$, is denoted by $\operatorname{Pr}\left(\omega^{\prime}\right)$.

The following definition was proposed in Roorda et al. (2004).

Definition 2.2 A multiperiod acceptability measure on the sequence space $\Omega$ is a mapping that assigns to each partial history $\omega^{\prime} \in \Omega^{\prime}$ an acceptability measure on $F\left(\omega^{\prime}\right)$.

The acceptability measure on $F\left(\omega^{\prime}\right)$ that is provided by a multiperiod acceptability measure $\phi$ will be denoted by $\phi\left(\cdot \mid \omega^{\prime}\right)$; the element of the real line associated by this mapping to a position $X$ on $F\left(\omega^{\prime}\right)$ is denoted by $\phi\left(X \mid \omega^{\prime}\right)$. When $X$ is a position on $\Omega$, we also write $\phi\left(X \mid \omega^{\prime}\right)$ instead of $\phi\left(\left.X\right|_{F\left(\omega^{\prime}\right)} \mid \omega^{\prime}\right)$. The situation at the initial time is represented by the sequence of zero length; instead of $\phi(X \mid 0)$, we also write $\phi(X)$. We say that a multiperiod

\footnotetext{
${ }^{3}$ Closure is understood here in the sense of the usual topology on $\mathbb{R}^{n}$. In more general settings where $\Omega$ is infinite, this topology needs to be replaced by an appropriate weak topology, cf. Delbaen (2002), Föllmer and Schied (2004).
} 
acceptability measure is coherent if all partial-information acceptability measures $\phi\left(\cdot \mid \omega^{\prime}\right)$ are coherent on $F\left(\omega^{\prime}\right)$.

The definition above defines a multiperiod acceptability measure as a family of restricted acceptability measures parametrized by partial histories $\omega^{\prime}$. Equivalently, one can consider the acceptability measures $\phi\left(\cdot \mid \omega^{\prime}\right)$ as being defined on the set of all positions $F(0)=\mathcal{X}(\Omega)$, if one adds the requirement that

$$
\phi\left(X \mid \omega^{\prime}\right)=\phi\left(\mathbb{1}_{F\left(\omega^{\prime}\right)} X \mid \omega^{\prime}\right)
$$

for all $X$ and all $\omega^{\prime}$. Indeed, the positions $X_{1}$ and $X_{2}$ are the same when restricted to $F\left(\omega^{\prime}\right)$ if and only if the equality $\mathbb{1}_{F\left(\omega^{\prime}\right)} X_{1}=\mathbb{1}_{F\left(\omega^{\prime}\right)} X_{2}$ holds. We call (2.2) the soundness property. ${ }^{4}$ An alternative phrasing of Def. 2.2 is therefore the following.

Definition 2.3 A multiperiod acceptability measure on the sequence space $\Omega$ is a mapping from $\mathcal{X}(\Omega) \times \Omega^{\prime}$ to $\mathbb{R}$ that satisfies the soundness property (2.2).

Below we often simply use the term "acceptability measure" rather than "multiperiod acceptability measure."

\subsection{Conditional evaluations}

Instead of considering a family of acceptability measures parametrized by sequences $\omega^{\prime}$ (which may be thought of as representing both current state and current time), one can also approach the notion of multiperiod acceptability by introducing a family of evaluation operators parametrized only by time. Specifically, for a mapping $\phi: \mathcal{X}(\Omega) \times \Omega^{\prime} \rightarrow \mathbb{R}$ and a given position $X$ one may introduce for each $t=0,1, \ldots, T$ the real-valued function $\mathcal{E}_{t}^{\phi}(X)$ defined on $\Omega$ by

$$
\mathcal{E}_{t}^{\phi}(X): \omega \mapsto \phi\left(X|\omega|_{t}\right)
$$

We also sometimes write $\mathcal{E}_{t}^{\phi} X$ (without brackets) instead of $\mathcal{E}_{t}^{\phi}(X)$. In this manner, one associates to any $\mathcal{F}_{T}$-measurable function $X$ an $\mathcal{F}_{t}$-measurable function $\mathcal{E}_{t}^{\phi}(X)$. Mappings of this type are generalizations of conditional expectation operators; they are called "conditional evaluations" by Peng (2004).

To a family of conditional evaluation operators $\left\{\mathcal{E}_{t}\right\}_{t}$, one can associate a mapping $\phi_{\mathcal{E}}$ : $\mathcal{X}(\Omega) \times \Omega^{\prime} \rightarrow \mathbb{R}$ defined by

$$
\phi_{\mathcal{E}}\left(X \mid \omega^{\prime}\right)=\mathcal{E}_{T\left(\omega^{\prime}\right)}(X)(\omega) \quad\left(\omega \succeq \omega^{\prime}\right) .
$$

\footnotetext{
${ }^{4}$ The notion of "regularity" introduced in Scandolo (2003, p. 32) is similar but not entirely the same; in particular Scandolo's notion implies that $\phi(0 \mid \cdot)=0$ whereas there is no such implication from the soundness property.
} 
This mapping is indeed unambiguously defined because of the requirement that $\mathcal{E}_{t}(X)$ must be $\mathcal{F}_{t}$-measurable. The two associations (2.3) and (2.4) establish a one-to-one relationship between conditional evaluation operators and mappings from $\mathcal{X}(\Omega) \times \Omega^{\prime}$ to $\mathbb{R}$. To ensure that the soundness property (2.2) holds, an additional condition has to be imposed. Such a condition is presented in the lemma below.

Lemma 2.4 Suppose that a family of operators $\left\{\mathcal{E}_{t}\right\}_{t}$ is given which map $\mathcal{F}_{T}$-measurable functions to $\mathcal{F}_{t}$-measurable functions. If this family is such that

$$
\mathcal{E}_{t}\left(\mathbb{1}_{F} X\right)=\mathbb{1}_{F} \mathcal{E}_{t}(X)
$$

for all $t \in\{0,1, \ldots, T\}, X \in \mathcal{X}(\Omega)$, and $F \in \mathcal{F}_{t}$, then the mapping $\phi_{\mathcal{E}}$ defined by (2.4) satisfies the soundness property (2.2).

Proof Take $\omega^{\prime} \in \Omega^{\prime}$ and $X \in \mathcal{X}(\Omega)$. For $\omega \succeq \omega^{\prime}$, we have

$$
\begin{aligned}
\phi_{\mathcal{E}}\left(\mathbb{1}_{F\left(\omega^{\prime}\right)} X \mid \omega^{\prime}\right) & =\mathcal{E}_{T\left(\omega^{\prime}\right)}\left(\mathbb{1}_{F\left(\omega^{\prime}\right)} X\right)(\omega) \\
& =\mathbb{1}_{F\left(\omega^{\prime}\right)}(\omega) \mathcal{E}_{T\left(\omega^{\prime}\right)}(X)(\omega) \\
& =\mathcal{E}_{T\left(\omega^{\prime}\right)}(X)(\omega) \\
& =\phi_{\mathcal{E}}\left(X \mid \omega^{\prime}\right) .
\end{aligned}
$$

The condition (2.5) is taken as an axiom for conditional evaluations by Peng (2004); see also Prop. 2.2.iv in Coquet et al. (2002). Within the context of a finite sample space $\Omega$, the condition may alternatively be formulated as follows.

Lemma 2.5 Consider a family $\left\{\mathcal{E}_{t}\right\}_{t}$ of evaluation operators satisfying $\mathcal{E}_{t}(0)=0$. The property (2.5) is satisfied for all $t, F \in \mathcal{F}_{t}$, and $X$ if and only if for all $\omega^{\prime}$ and all $X$ the following holds:

$$
\mathcal{E}_{T\left(\omega^{\prime}\right)}\left(\mathbb{1}_{F\left(\omega^{\prime}\right)} X\right)=\mathbb{1}_{F\left(\omega^{\prime}\right)} \mathcal{E}_{T\left(\omega^{\prime}\right)}(X) .
$$

Proof It is clear that (2.5) implies (2.6). Assume now that (2.6) holds for all $\omega^{\prime}$. Take $t \in\{0, \ldots, T\}, G \in \mathcal{F}_{t}$, and $\omega \in \Omega$. Define $\omega^{\prime}=\left.\omega\right|_{t}$. If $\omega \in G$, then in fact $F\left(\omega^{\prime}\right) \subset G$ and so we can write

$$
\begin{aligned}
\mathcal{E}_{t}\left(\mathbb{1}_{G} X\right)(\omega) & =\mathbb{1}_{F\left(\omega^{\prime}\right)}(\omega) \mathcal{E}_{t}\left(\mathbb{1}_{G} X\right)(\omega) \\
& =\mathcal{E}_{t}\left(\mathbb{1}_{F\left(\omega^{\prime}\right)} \mathbb{1}_{G} X\right)(\omega) \\
& =\mathcal{E}_{t}\left(\mathbb{1}_{F\left(\omega^{\prime}\right)} X\right)(\omega) \\
& =\mathbb{1}_{F\left(\omega^{\prime}\right)}(\omega) \mathcal{E}_{t}(X)(\omega) \\
& =\mathcal{E}_{t}(X)(\omega) .
\end{aligned}
$$


On the other hand, if $\omega \notin G$, then $F\left(\omega^{\prime}\right) \cap G=\emptyset$, and therefore the same reasoning as above shows in this case that $\mathcal{E}_{t}\left(\mathbb{1}_{G} X\right)(\omega)=\mathcal{E}_{t}(0)(\omega)=0$. So in both cases we find

$$
\mathcal{E}_{t}\left(\mathbb{1}_{G} X\right)(\omega)=\mathbb{1}_{G}(\omega) \mathcal{E}_{t}(X)(\omega)
$$

which shows that (2.5) holds.

The definition (2.3) can be extended to stopping times in the obvious way. Moreover, we have, for any stopping time $\tau$,

$$
\mathcal{E}_{\tau}^{\phi}(X)=\Sigma_{\omega^{\prime} \in \Omega_{\tau}} \phi\left(X \mid \omega^{\prime}\right) \mathbb{1}_{F\left(\omega^{\prime}\right)}
$$

Here the right hand side can be viewed as the position that is obtained by replacing, at each stopping node $\omega^{\prime}$, the position $\left.X\right|_{F\left(\omega^{\prime}\right)}$ by its certainty equivalent $\phi\left(X \mid \omega^{\prime}\right)$.

In this paper we work mainly with formulations in terms of acceptability measures, but it is seen from the above that equivalent formulations in terms of conditional evaluation operators can always be given.

\section{Consistency conditions}

In this section we introduce a number of different notions of time consistency. The concepts that we use were already described briefly in the Introduction.

Definition 3.1 A multiperiod acceptability measure $\phi$ is said to be conditionally consistent if, for all positions $X$ and for all partial histories $\omega^{\prime} \in \Omega^{\prime}$, we have $\phi\left(X \mid \omega^{\prime}\right) \geq 0$ if and only if $\phi\left(\mathbb{1}_{F\left(\omega^{\prime}\right)} X\right) \geq 0$.

Definition 3.2 A multiperiod acceptability measure $\phi$ is said to be sequentially consistent if, whenever the relation $\phi\left(X \mid \omega^{\prime}\right) \geq 0$ holds for some position $X$ and some partial history $\omega^{\prime} \in \Omega^{\prime}$, there exists a full history $\omega \in F\left(\omega^{\prime}\right)$ such that $\phi\left(X|\omega|_{t}\right) \geq 0$ for $t=T\left(\omega^{\prime}\right), \ldots, T$, and the same conclusion holds with the direction of the inequality reversed when $\phi\left(X \mid \omega^{\prime}\right) \leq$ 0 .

Definition 3.3 A multiperiod acceptability measure $\phi$ is said to satisfy the tower law of conditional evaluations if for all positions $X$, all partial histories $\omega^{\prime}$, and all stopping times $\tau \geq T\left(\omega^{\prime}\right)$ we have $\phi\left(X \mid \omega^{\prime}\right)=\phi\left(\mathcal{E}_{\tau}^{\phi} X \mid \omega^{\prime}\right)$.

Definition 3.4 A multiperiod acceptability measure $\phi$ is said to be dynamically consistent if, for all partial histories $\omega^{\prime} \in \Omega^{\prime}$ and for all positions $X$ and $Y$ such that $\phi\left(X \mid \omega^{\prime} \alpha\right)=$ $\phi\left(Y \mid \omega^{\prime} \alpha\right)$ for all $\alpha \in A$, we have $\phi\left(X \mid \omega^{\prime}\right)=\phi\left(Y \mid \omega^{\prime}\right)$. 
Versions of the notion of dynamic consistency have been used extensively in the recent literature on dynamic acceptability measures, see for instance Artzner et al. (2004; Definition 5.2), Frittelli and Gianin (2004; Definition 18). It was shown in Roorda et al. (2004, Prop. 2.6) that for coherent risk measures the notion of dynamic consistency is equivalent to the property of "stepwise monotonicity" that is defined as follows:

$$
\text { if } \phi\left(X \mid \omega^{\prime} \alpha\right) \geq \phi\left(Y \mid \omega^{\prime} \alpha\right) \text { for all } \alpha \in A \text {, then } \phi\left(X \mid \omega^{\prime}\right) \geq \phi\left(Y \mid \omega^{\prime}\right) \text {. }
$$

The tower law as formulated above may also be written in the form $\mathcal{E}_{\tau_{1}} \mathcal{E}_{\tau_{2}} X=\mathcal{E}_{\tau_{1}} X$ for stopping times $\tau_{1} \leq \tau_{2}$, which makes it an obvious extension of the tower law of conditional expectations. The notions of conditional and sequential consistency do not seem to have been formally defined before in the literature. Sequential consistency expresses the requirement that an initially acceptable position remains acceptable along at least one full path $\omega \in \Omega .{ }^{5}$ Conditional consistency requires that a conditional version of a payoff is initially acceptable if and only if it is acceptable at the moment the condition would become reality. An alternative phrasing might be that conditioning in terms of payoff has the same effect as conditioning in terms of information.

A natural requirement to impose is that a given acceptability measure should be sensitive at least to some degree to each of the scenarios in a given model. This requirement can be formulated as follows:

$$
\phi\left(X \mid \omega^{\prime}\right)<0 \text { for all } \omega^{\prime} \in \Omega^{\prime} \text { and } X \leq 0 \text { such that } \mathbb{1}_{F\left(\omega^{\prime}\right)} X \neq 0
$$

This condition corresponds to the axiom of relevance in Artzner et al. (1999). ${ }^{6}$ A slightly stronger version is:

$$
\phi\left(X \mid \omega^{\prime}\right)>0 \text { for all } \omega^{\prime} \in \Omega^{\prime} \text { and } X \geq 0 \text { such that } \mathbb{1}_{F\left(\omega^{\prime}\right)} X \neq 0 .
$$

We refer to this property as strong relevance; it corresponds to the relevance condition in Föllmer and Schied (2002, Definition 4.27). For coherent measures we always have $\phi(X) \leq$ $-\phi(-X)$ so that strong relevance indeed implies relevance.

\footnotetext{
${ }^{5}$ It is easily verified that, assuming the translation property, the non-strict inequalities in the definition can be replaced by a strict inequalities without effect, and hence the same interpretation is valid for initially unacceptable positions.

${ }^{6}$ A given scenario set can be adapted as follows to satisfy the relevance condition. Instead of $\Omega$, consider the set of initially relevant states $\Omega_{0}:=\left\{\omega \in \Omega \mid \phi\left(-\mathbb{1}_{\omega}\right)<0\right\}$. Under the assumption that these states remain relevant, i.e., $\phi\left(-\mathbb{1}_{\omega}|\omega|_{t}\right)<0$ for all $\omega \in \Omega_{0}$ and $t>0$, the condition (3.2) holds on $\Omega_{0}$, and the restriction of $\Omega$ to $\Omega_{0}$ only involves some obvious adaptations of notation.
} 


\section{Single-step formulations}

In a discrete-time setting it is a natural idea to specify consistency in terms of single-step conditions; this would correspond to infinitesimal characterizations in a continuous-time framework. We provide single-step characterizations for all notions of time consistency that were introduced in the previous section. The proofs of the theorems in this section are given in the Appendix.

Our first result shows that the time consistency property expressed by the tower law of conditional evaluations is in fact the same as dynamic consistency. Condition 1 in the theorem below furthermore provides a recursive way of computing evaluations under dynamically consistent acceptability measures.

TheOREM 4.1 Let $\phi$ be a coherent multiperiod acceptability measure that satisfies the relevance condition. The measure $\phi$ satisfies the tower law of conditional evaluations if and only if the following equivalent conditions hold.

1. $\phi\left(X \mid \omega^{\prime}\right)=\phi\left(\mathcal{E}_{T\left(\omega^{\prime}\right)+1}^{\phi} X \mid \omega^{\prime}\right)$ for all $X \in \mathcal{X}(\Omega), \omega^{\prime} \in \Omega^{\prime \prime}$.

2. $\phi$ is dynamically consistent.

The next result concerns sequential consistency (Def. 3.2). Condition 4 in the theorem below immediately implies that any dynamically consistent acceptability measure that satisfies the relevance condition is sequentially consistent.

TheOrem 4.2 Let $\phi$ be a coherent multiperiod acceptability measure that satisfies the relevance condition. The measure $\phi$ is sequentially consistent if and only if the following equivalent conditions hold.

1. For all $X \in \mathcal{X}(\Omega), \omega^{\prime} \in \Omega^{\prime \prime}$, the following relations are valid:

(a) $\phi\left(X \mid \omega^{\prime}\right) \geq 0$ only if there exists $\alpha \in A$ such that $\phi\left(X \mid \omega^{\prime} \alpha\right) \geq 0$

(b) $\phi\left(X \mid \omega^{\prime}\right) \leq 0$ only if there exists $\alpha \in A$ such that $\phi\left(X \mid \omega^{\prime} \alpha\right) \leq 0$.

2. $\phi\left(X \mid \omega^{\prime}\right) \in\left[\min _{\alpha \in A} \phi\left(X \mid \omega^{\prime} \alpha\right), \max _{\alpha \in A} \phi\left(X \mid \omega^{\prime} \alpha\right)\right]$.

3. $\phi$ satisfies the following implication: $\phi\left(X \mid \omega^{\prime} \alpha\right)=0$ for all $\alpha \in A \Rightarrow \phi\left(X \mid \omega^{\prime}\right)=0$.

4. $\phi$ satisfies (3.1) for all pairs $X, Y$ with $X=0$ or $Y=0$.

The third main result of this section provides single-step characterizations for conditional consistency (Def. 3.1). Condition 2 below can be viewed as a confirmation rule: initially (un)acceptable positions associated to a future event become even more (un)acceptable when that event does take place. 
THEOREM 4.3 Let $\phi$ be a coherent multiperiod acceptability measure that satisfies the relevance condition. The measure $\phi$ is conditionally consistent if and only if the following equivalent conditions hold for all $X \in \mathcal{X}(\Omega)$ and $\omega^{\prime} \in \Omega^{\prime \prime}$.

1. For all $\alpha \in A, \phi\left(X \mid \omega^{\prime} \alpha\right) \geq 0 \Leftrightarrow \phi\left(\mathbb{1}_{F\left(\omega^{\prime} \alpha\right)} X \mid \omega^{\prime}\right) \geq 0$.

2. For all $\alpha \in A, \phi\left(\mathbb{1}_{F\left(\omega^{\prime} \alpha\right)} X \mid \omega^{\prime}\right) \in \operatorname{cch}\left\{0, \phi\left(X \mid \omega^{\prime} \alpha\right)\right\}$ (the closed interval with endpoints 0 and $\left.\phi\left(X \mid \omega^{\prime} \alpha\right)\right)$, and $\phi\left(\mathbb{1}_{F\left(\omega^{\prime} \alpha\right)} X \mid \omega^{\prime}\right)=0$ implies $\phi\left(X \mid \omega^{\prime} \alpha\right) \geq 0$.

If $\phi$ is strongly relevant, the second condition in item 2 may be replaced by the following condition: $\phi\left(\mathbb{1}_{F\left(\omega^{\prime} \alpha\right)} X \mid \omega^{\prime}\right)=0$ implies $\phi\left(X \mid \omega^{\prime} \alpha\right)=0$.

For future reference we mention the following implication of conditional consistency.

LEMma 4.4 Let $\phi$ be a coherent multiperiod acceptability measure that satisfies the relevance condition. If $\phi$ is conditionally consistent, then

$$
\phi\left(X \mid \omega^{\prime}\right) \geq \min _{\alpha \in A} \phi\left(X \mid \omega^{\prime} \alpha\right)
$$

Proof We have to prove that $\phi\left(X \mid \omega^{\prime}\right)-c_{\min } \geq 0$ with $c_{\min }:=\min _{\alpha \in A} \phi\left(X \mid \omega^{\prime} \alpha\right)$, if $\phi$ is conditionally consistent. From superadditivity of $\phi$, it follows that $\phi\left(X \mid \omega^{\prime}\right)-c_{\min }=$ $\phi\left(X-c_{\min } \mid \omega^{\prime}\right)=\phi\left(\Sigma_{\alpha \in A} \mathbb{1}_{F\left(\omega^{\prime} \alpha\right)}\left(X-c_{\min }\right) \mid \omega^{\prime}\right) \geq \Sigma_{\alpha \in A} \mathbb{1}_{F\left(\omega^{\prime} \alpha\right)} \phi\left(\left(X-c_{\min }\right) \mid \omega^{\prime}\right)$, and according to Condition 1 in Theorem 4.3 , each term $\phi\left(\mathbb{1}_{F\left(\omega^{\prime} \alpha\right)}\left(X-c_{\min }\right) \mid \omega^{\prime}\right)$ is nonnegative if and only if $\phi\left(X-c_{\min } \mid \omega^{\prime} \alpha\right) \geq 0$, which indeed holds true for all $\alpha \in A$.

So conditional consistency implies the lower bound in Theorem 4.2.2 for $\phi\left(X \mid \omega^{\prime}\right)$. A similar observation is made in Artzner et al. (2004; remark following Definition 5.2).

\section{Relations between consistency types}

One of the main purposes of this paper is to show that, under mild conditions, the tower law of conditional evaluations implies sequential consistency, and sequential consistency in turn implies conditional consistency. The precise formulation reads as follows.

THEOREM 5.1 The following implication relations hold for properties satisfied by coherent multiperiod acceptability measures. Under the relevance condition (3.2), the tower law of conditional evaluations entails both sequential and conditional consistency. Under the strong relevance condition (3.3), sequential consistency implies conditional consistency.

The proof is given in the Appendix. Here we discuss a few examples that demonstrate differences between the various notions of consistency. Some notation will be required. In all examples, we consider two-period binomial trees, so that, with $A=\{u, d\}$, we have 
$\Omega=\{u u, u d, d u, d d\}$. Suppose that numbers $p_{i} \in[0,1]$ have been given. Let $\mathbb{P}_{i}$ denote the probability measure on $A$ that assigns probability $p_{i}$ to event $u$ and probability $1-p_{i}$ to event $d$. Denote by $\mathbb{P}_{i_{k}^{j}}$ the probability measure on $\Omega$ that is obtained by making the first step subject to $\mathbb{P}_{i}$, and the second step subject to $\mathbb{P}_{j}$ conditional on $u$ and to $\mathbb{P}_{k}$ conditional on $d$, so that for instance the probability of $u u$ is $p_{i} p_{j}$ and the probability of $d d$ is $\left(1-p_{i}\right)\left(1-p_{k}\right)$. The position $X \in \mathcal{X}(\Omega)$ with payoffs $X(u u)=x_{1}, X(u d)=x_{2}$, $X(d u)=x_{3}, X(d d)=x_{4}$ is denoted by $\left[\begin{array}{llll}x_{1} & x_{2} & x_{3} & x_{4}\end{array}\right]$. In view of the representation theorem (Thm. 2.1) and the definition of multiperiod acceptability measures as families of restricted acceptability measures, a coherent multiperiod acceptability measure on the twoperiod binomial tree may be given by specifying three collections of probability measures $\mathcal{P}_{\omega^{\prime}}$ on $F\left(\omega^{\prime}\right)$ for $\omega^{\prime}=0, u, d$. We write $\phi\left(\cdot \mid \omega^{\prime}\right) \sim \mathcal{P}_{\omega^{\prime}}$. In all examples except the last one, we take $p_{1}=0.6, p_{2}=0.4$. As far as consistency claims in the examples below are not proved explicitly, they follow easily from the characterizations of the next section.

EXAmPLE 5.2 First, we present an example to show that a coherent multiperiod acceptability measure need not be conditionally consistent. Take $X=\left[\begin{array}{llll}-1 & 1 & 0 & 0\end{array}\right]$. Define $\phi$ by

$$
\phi(\cdot \mid 0) \sim\left\{\mathbb{P}_{1_{1}^{1}}\right\}, \quad \phi(\cdot \mid u) \sim\left\{\mathbb{P}_{2}\right\}, \phi(\cdot \mid d) \sim\left\{\mathbb{P}_{2}\right\} .
$$

We then have $\phi(X \mid u)=0.2$ and $\phi\left(\mathbb{1}_{F(u)} X\right)=-0.12$, so that conditional consistency does not hold. Under $\phi$, the payoff " $X$ if $u$ occurs" is not acceptable initially, but after $u$ does occur, the payoff $X$ becomes acceptable.

EXAMPLE 5.3 Our second example shows a conditionally but not sequentially consistent acceptability measure. This example is taken from Roorda et al. (2004). Define $\phi$ by

$$
\phi(\cdot \mid 0) \sim\left\{\mathbb{P}_{1_{1}^{1}}, \mathbb{P}_{2_{2}^{2}}\right\}, \quad \phi(\cdot \mid u) \sim\left\{\mathbb{P}_{1}, \mathbb{P}_{2}\right\}, \quad \phi(\cdot \mid d) \sim\left\{\mathbb{P}_{1}, \mathbb{P}_{2}\right\}
$$

This measure is conditionally consistent. To see that it is not sequentially consistent, take $X=\left[\begin{array}{llll}-44 & 56 & 56 & -44\end{array}\right]$. We then have $\phi(X)=4$, while $\phi(X \mid u)=\phi(X \mid d)=-4$.

EXAMPLE 5.4 The measure $\phi$ given by

$$
\phi(\cdot \mid 0) \sim\left\{\mathbb{P}_{1_{1}^{1}}, \mathbb{P}_{1_{2}^{1}}, \mathbb{P}_{2_{1}^{2}}, \mathbb{P}_{2_{2}^{2}}\right\}, \phi(\cdot \mid u) \sim\left\{\mathbb{P}_{1}, \mathbb{P}_{2}\right\}, \phi(\cdot \mid d) \sim\left\{\mathbb{P}_{1}, \mathbb{P}_{2}\right\}
$$

is sequentially consistent, but it does not satisfy the tower law of conditional evaluations. To see that the tower law does not hold, consider the position $X=\left[\begin{array}{llll}-1 & 4 & 0 & 0\end{array}\right]$. The conditional evaluation of this position at time 1 is given by $\phi(X \mid u)=1$ and $\phi(X \mid d)=0$, so its certainty equivalent may be described by $X^{\prime}=\left[\begin{array}{llll}1 & 1 & 0 & 0\end{array}\right]$. We have $\phi(X \mid 0)=0.6$ (the minimum is reached for test measures $\mathbb{P}_{1_{1}^{1}}$ and $\mathbb{P}_{1_{2}^{1}}$ ), while $\phi\left(X^{\prime} \mid 0\right)=0.4$ (the minimum is reached for test measures $\mathbb{P}_{2_{1}^{2}}$ and $\mathbb{P}_{2_{2}^{2}}$ ). 
EXAMPLE 5.5 Finally, an example is given of a sequentially consistent but not conditionally consistent acceptability measure. In this example, the relevance axiom is not satisfied. Take $p_{3}=0$. Define $\phi$ by

$$
\phi(\cdot \mid 0) \sim\left\{\mathbb{P}_{1_{1}^{1}}, \mathbb{P}_{3_{1}^{3}}\right\}, \phi(\cdot \mid u) \sim\left\{\mathbb{P}_{1}, \mathbb{P}_{3}\right\}, \phi(\cdot \mid d) \sim\left\{\mathbb{P}_{1}\right\}
$$

For $X=\left[\begin{array}{lll}1 & -1 & 0\end{array}\right], \phi\left(\mathbb{1}_{F(u)} X\right)=\min (0,0.12)=0$, while $\phi(X \mid u)=-1$, so conditional consistency does not hold. Sequential consistency is satisfied, however; this can be seen as follows. If $\phi(X) \geq 0$, then $E_{\mathbb{P}_{3}^{3}} X \geq 0$ so that (writing $X_{\alpha}$ for $X$ restricted to $F(\alpha)$ ) $\phi(X \mid d)=E_{\mathbb{P}_{1}}\left[X_{d}\right] \geq 0$. If $\phi(X) \leq 0$, then $E_{\mathbb{P}_{1_{1}^{1}}} X \leq 0$ or $E_{\mathbb{P}_{3_{1}^{3}}} X \leq 0$, so that $0.6 E_{\mathbb{P}_{1}}\left[X_{u}\right]+$ $0.4 E_{\mathbb{P}_{1}}\left[X_{d}\right] \leq 0$ or $E_{\mathbb{P}_{1}}\left[X_{d}\right] \leq 0$. In the latter case, it follows immediately that $\phi(X \mid d) \leq$ 0 . In the former case, if $\phi(X \mid d)>0$ we must have $E_{\mathbb{P}_{1}}\left[X_{u}\right]<0$ so that $\phi(X \mid u)=$ $\min \left(E_{\mathbb{P}_{1}}\left[X_{u}\right], E_{\mathbb{P}_{3}}\left[X_{u}\right]\right)<0$.

\section{Representation by collections of probability measures}

It follows from the representation theorem quoted above (Thm. 2.1) that any coherent multiperiod acceptability measure $\phi$ can be represented in the form

$$
\phi\left(X \mid \omega^{\prime}\right)=\inf _{\mathbb{P} \in \mathcal{P}_{\omega^{\prime}}} E_{\mathbb{P}}\left[\left.X\right|_{F\left(\omega^{\prime}\right)}\right]
$$

where, for each $\omega^{\prime} \in \Omega^{\prime}, \mathcal{P}_{\omega^{\prime}}$ is a nonempty collection of probability measures on $F\left(\omega^{\prime}\right)$. A collection of probability measures that is parametrized in this way by partial histories will be referred to as a test set family and generally denoted by $\mathcal{T}$. Being collections of collections of probability measures, test set families are somewhat complicated objects. This is one of the motivations to consider simpler and more restricted representation forms. In this section we discuss such representation forms and associated properties of test set families.

The following terminology and notation will be used. The probability that $\omega$ occurs is denoted by $\mathbb{P}(\omega)$. The marginal probability of a partial history $\omega^{\prime} \in \Omega^{\prime}$ is given, with some (further) abuse of notation, by

$$
\mathbb{P}\left(\omega^{\prime}\right)=\sum_{\omega^{\prime} \preceq \omega} \mathbb{P}(\omega)=\mathbb{P}\left(F\left(\omega^{\prime}\right)\right) .
$$

The conditional probability, given a partial history $\omega^{\prime} \in \Omega^{\prime}$ with $\mathbb{P}\left(\omega^{\prime}\right)>0$, of a sequence $\omega \succeq \omega^{\prime}$ is given by

$$
\mathbb{P}\left(\omega \mid \omega^{\prime}\right)=\frac{\mathbb{P}(\omega)}{\mathbb{P}\left(\omega^{\prime}\right)} .
$$

We will also need the "single-step conditional probabilities" defined by

$$
\mathbb{P}^{\mathrm{s}}\left(\alpha \mid \omega^{\prime}\right)=\frac{\mathbb{P}\left(\omega^{\prime} \alpha\right)}{\mathbb{P}\left(\omega^{\prime}\right)} .
$$


Given a collection $\mathcal{P}$ of probability measures on $\Omega$, there is an induced collection, denoted by $\mathcal{P}^{\mathrm{s}}\left(\omega^{\prime}\right)$, of single-step conditional probabilities at a given partial history $\omega^{\prime} \in \Omega^{\prime \prime}$ :

$$
\mathcal{P}^{\mathrm{s}}\left(\omega^{\prime}\right)=\left\{\mathbb{P}^{\mathrm{s}}\left(\cdot \mid \omega^{\prime}\right) \mid \mathbb{P} \in \mathcal{P} \text { with } \mathbb{P}\left(\omega^{\prime}\right)>0\right\} .
$$

Likewise, at each $\omega^{\prime} \in \Omega^{\prime \prime}$ there is an induced collection of probability measures on $F\left(\omega^{\prime}\right)$ :

$$
\mathcal{P}\left(\omega^{\prime}\right):=\left\{\mathbb{P}\left(\cdot \mid \omega^{\prime}\right) \mid \mathbb{P} \in \mathcal{P} \text { with } \mathbb{P}\left(\omega^{\prime}\right)>0\right\} .
$$

It should be noted that even when $\mathcal{P}$ is a closed and convex collection of probability measures on $\Omega$, the induced collection $\mathcal{P}\left(\omega^{\prime}\right)$ of conditional measures at a given node $\omega^{\prime}$ need not be closed, and the same is true for the induced collection $\mathcal{P}^{\mathrm{s}}\left(\omega^{\prime}\right)$ of single-period probability measures. This is demonstrated in the following simple example.

EXAmple 6.1 Consider a two-period binomial tree. Define a collection of probability measures on $\Omega=\{u u, u d, d u, d d\}$ by

$$
\mathcal{P}=\{\mathbb{P} \mid \exists p \in[0,1] \text { s.t. } \mathbb{P}(u)=\mathbb{P}(u \mid u)=\mathbb{P}(u \mid d)=p\} .
$$

The set $\mathcal{P}$ is closed; however, the induced collection $\mathcal{P}(u)$ of probability measures on $\mathcal{F}(u)$ consists of the measures that assign positive probability to the event $u$, and consequently $\mathcal{P}(u)$ is not closed.

We say that a collection $\mathcal{P}$ of probability measures on $\Omega$ is fully supported if for each $\omega \in \Omega$ there is at least one $\mathbb{P} \in \mathcal{P}$ such that $\mathbb{P}(\omega)>0$. From such a collection one can form a test set family by the rule

$$
\mathcal{T}(\mathcal{P})=\left\{\mathcal{P}\left(\omega^{\prime}\right)\right\}_{\omega^{\prime} \in \Omega^{\prime}}
$$

This provides a way to specify a test set family just by means of specification of a collection of probability measures on $\Omega$. The associated multiperiod acceptability measure is denoted by $\phi_{\mathcal{P}}$; we have

$$
\phi_{\mathcal{P}}\left(X \mid \omega^{\prime}\right)=\inf _{\mathbb{P} \in \mathcal{P}, \mathbb{P}\left(\omega^{\prime}\right)>0} E_{\mathbb{P}}\left[X \mid \omega^{\prime}\right]
$$

for positions $X$ and partial histories $\omega^{\prime}$.

Definition 6.2 A multiperiod acceptability measure $\phi$ is said to be globally representable if it admits a representation of the form (6.9), i.e. if there exists a fully supported collection $\mathcal{P}$ of probability measures on $\Omega$ such that $\phi=\phi_{\mathcal{P}}$. The collection $\mathcal{P}$ is called a global test set of $\phi$.

If there is no danger of confusion, we will sometimes speak of just a "test set" rather than a "global test set." Only acceptability measures that are relevant in the sense of (3.2) can be globally representable; compare Artzner et al. (1999, Remark 4.3). 
A specific way to specify a global test set is the following. Suppose that one provides, at each node $\omega^{\prime}$, a collection of probability measures on the event set $A$. Let these collections of single-step probabilities be denoted by $\mathcal{P}_{\omega^{\prime}}^{\text {s }}$ for $\omega^{\prime} \in \Omega^{\prime \prime}$. Then one can define a test set $\mathcal{P}$ by

$$
\mathcal{P}=\left\{\mathbb{P} \in \operatorname{Pr} \mid \text { for all } \omega^{\prime} \in \Omega^{\prime \prime}: \mathbb{P}^{\mathrm{s}}\left(\cdot \mid \omega^{\prime}\right) \in \mathcal{P}_{\omega^{\prime}}^{\mathrm{s}}\right\}
$$

In a test set formed in this way, probabilities associated to full histories $\omega \succeq \omega^{\prime}$ by test measures at a given node $\omega^{\prime}$ are obtained as products of single-step probabilities at nodes intermediate between $\omega^{\prime}$ and $\omega$. Test sets of this type are known by various names in the literature; the terminology in the definition below follows Roorda et al. (2004). Note that if a test set is constructed as in (6.10), then necessarily $\mathcal{P}_{\omega^{\prime}}^{\mathrm{s}}=\mathcal{P}^{\mathrm{s}}\left(\omega^{\prime}\right)$ for all $\omega^{\prime} \in \Omega^{\prime \prime}$. The definition may therefore be stated as follows.

Definition 6.3 A test set $\mathcal{P}$ on a sequence space $\Omega$ is said to be of product type if

$$
\left\{\mathbb{P} \in \operatorname{Pr} \mid \text { for all } \omega^{\prime} \in \Omega^{\prime \prime}: \mathbb{P}^{\mathrm{s}}\left(\cdot \mid \omega^{\prime}\right) \in \mathcal{P}^{\mathrm{s}}\left(\omega^{\prime}\right)\right\}=\mathcal{P} .
$$

The final property of test sets that we need is based on the relation between conditional and joint distributions. This may be discussed in a more general context first. If we have two random variables $V$ and $W$ both taking only finitely many values, then the joint distribution of $V$ and $W$ is related to the conditional distributions of $W$ given $V$ by

$$
\mathbb{P}(V=i, W=j)=\mathbb{P}(V=i) \mathbb{P}(W=j \mid V=i) .
$$

Conversely, given a collection of probability distributions $\mathbb{P}_{i}$ for the variable $W$, one might consider the set of all joint distributions that are related to the given collection by means of the rule above. Obviously, this set is parametrized by the set of all probability measures that may be placed on the conditioning variable $V$. This observation gives rise to the following definition, in which we adapt the notation to some extent to the intended application. Given a finite set $\Omega$ and a collection $\left\{\mathbb{P}_{\alpha}\right\}_{\alpha \in A}$ of probability measures on $\Omega$, where $A$ is another finite set, we will say that a probability distribution $\mathbb{P}$ on $A \times \Omega$ is a junct of the collection $\left\{\mathbb{P}_{\alpha}\right\}_{\alpha \in A}$ if there exists a probability distribution $\tilde{\mathbb{P}}$ on $A$ such that for all $\alpha \in A$ and $\omega \in \Omega$ we have

$$
\mathbb{P}(\alpha, \omega)=\tilde{\mathbb{P}}(\alpha) \mathbb{P}_{\alpha}(\omega)
$$

Note that the definition allows that $\tilde{\mathbb{P}}(\alpha)=0$ for some $\alpha$. We now return to the specific framework of this paper. 
Definition 6.4 A family $\left\{\mathcal{P}_{\omega^{\prime}}\right\}_{\omega^{\prime} \in \Omega^{\prime}}$ of collections of probability measures is said to be juncted at $\omega^{\prime} \in \Omega^{\prime \prime}$ if each selection $\left\{\mathbb{P}_{\alpha}\right\}_{\alpha \in A}$ with $\mathbb{P}_{\alpha} \in \mathcal{P}_{\omega^{\prime} \alpha}$ admits a junct in $\mathcal{P}_{\omega^{\prime}}$. The family is said to be juncted if it is juncted at every $\omega^{\prime} \in \Omega^{\prime \prime}$.

The expectation operator $E_{\mathbb{P}}$ associated to a probability measure $\mathbb{P} \in \operatorname{Pr}\left(\omega^{\prime}\right)$ is an operator on $\mathcal{X}\left(F\left(\omega^{\prime}\right)\right)$; we can extend it to an operator on $\mathcal{X}(\Omega)$, still denoted by $E_{\mathbb{P}}$, by defining

$$
E_{\mathbb{P}} X=E_{\mathbb{P}}\left[\left.X\right|_{F\left(\omega^{\prime}\right)}\right]=\sum_{\omega \succeq \omega^{\prime}} \mathbb{P}(\omega) X(\omega) \quad(X \in \mathcal{X}(\Omega)) .
$$

In terms of this notation, the property of junctedness may alternatively be formulated as follows: for each selection $\left\{\mathbb{P}_{\alpha}\right\}_{\alpha \in A}$ with $\mathbb{P}_{\alpha} \in \mathcal{P}_{\omega^{\prime} \alpha}$, there exists $\mathbb{P} \in \mathcal{P}_{\omega^{\prime}}$ such that

$$
E_{\mathbb{P}} \in \operatorname{ch}\left\{E_{\mathbb{P}_{\alpha}}\right\}_{\alpha \in A} .
$$

Comparing the notion of junctedness to the product property, we see that the latter property implies that at every node $\omega^{\prime} \in \Omega^{\prime \prime}$ there is a nonempty collection of marginal (i.e. single-step) distributions such that the combination of any of these with any selection of conditional distributions (i.e. elements of $\mathcal{P}_{\omega^{\prime} \alpha}$ ) gives rise to a simultaneous distribution that belongs to $\mathcal{P}_{\omega^{\prime}}$. Junctedness means that for any selection of conditionals there is at least one marginal such that the induced simultaneous distribution belongs to $\mathcal{P}_{\omega^{\prime}}$, but in general different marginals may be used for different selections. The product property may therefore be viewed as a uniform version of junctedness. In particular, any product-type test set family is juncted.

It has already been noted above that conditioning in general does not preserve closedness of test sets. Therefore we define junctedness for global test sets as follows.

Definition 6.5 A global test set $\mathcal{P} \subset \operatorname{Pr}$ is said to be juncted if the family $\overline{\mathcal{T}}(\mathcal{P}):=$ $\left\{\overline{\mathcal{P}\left(\omega^{\prime}\right)}\right\}_{\omega^{\prime} \in \Omega^{\prime}}$ is juncted.

The following example shows that the presence of the closure operator in the above definition does indeed make a difference.

EXAmple 6.6 Consider a three-period binomial tree with single-step event set $A=\{u, d\}$. Let $\mathcal{P}$ be the collection of all probability measures on $\Omega$ that satisfy

$$
\mathbb{P}(u \mid u u)+\mathbb{P}(u \mid u d) \geq 2 \mathbb{P}(u)
$$

and

$$
\mathbb{P}\left(u \mid \omega^{\prime}\right)=\mathbb{P}\left(d \mid \omega^{\prime}\right)=\frac{1}{2} \quad \text { for } \omega^{\prime}=u, d, d u, d d .
$$

Clearly, the collection $\mathcal{P}$ is closed. The convexity of $\mathcal{P}$ is seen by rewriting the requirement (6.16) in terms of unconditional probabilities as follows:

$$
\mathbb{P}(\text { иии })+\mathbb{P}(u d u) \geq(\mathbb{P}(u))^{2} .
$$


The set described by this inequality is convex, since it is a sublevel set of a convex function. The constraints added by (6.17) are linear and so they preserve convexity. It can be verified that the collection $\mathcal{P}$ is juncted according to Def. 6.5. Note that the collections of conditional test measures $\mathcal{P}(u u)$ and $\mathcal{P}(u d)$ both consist simply of all probability measures on the set $\{u, d\}$. Select the measures $\mathbb{P}_{1} \in \mathcal{P}(u u)$ and $\mathbb{P}_{2} \in \mathcal{P}(u d)$ that assign zero probability to $u$. Since the collection of conditional measures $\mathcal{P}(u)$ is described by

$$
\mathbb{P}(u \mid u)=\mathbb{P}(d \mid u)=\frac{1}{2}, \quad \mathbb{P}(u u \mid u)+\mathbb{P}(d u \mid u)>0
$$

the selection $\left(\mathbb{P}_{1}, \mathbb{P}_{2}\right)$ does not admit a junct in $\mathcal{P}(u)$. Therefore, the family $\left\{\mathcal{P}\left(\omega^{\prime}\right)\right\}_{\omega^{\prime} \in \Omega^{\prime}}$ is not juncted.

\section{Characterizations in terms of test sets}

The purpose of this section is to establish connections between the notions of consistency that were introduced in Section 3 and the representation forms discussed in the previous section. The main results are summarized in the theorem below. We use the notion of global representability (Def.6.2) and other definitions of the previous section.

TheOREM 7.1 Let $\phi$ be a coherent multiperiod acceptability measure that satisfies the relevance condition (3.2).

1. The measure $\phi$ is conditionally consistent if and only if it can be globally represented.

2. The measure $\phi$ is conditionally and sequentially consistent if and only if it can be globally represented by a juncted test set.

3. The measure $\phi$ satisfies the tower law property if and only if it can be globally represented by a test set of product type.

\section{ProOF}

\section{Claim 1.}

Concerning the "if" part, assume $\phi=\phi_{\mathcal{P}}$ as defined in (6.9). In particular, $\mathcal{P}$ represents $\phi(\cdot \mid 0)$, cf. (6.1), and hence

$$
\begin{aligned}
\phi\left(\mathbb{1}_{F\left(\omega^{\prime}\right)} X\right) \geq 0 & & \Leftrightarrow \\
E_{\mathbb{P}}\left[\mathbb{1}_{F\left(\omega^{\prime}\right)} X\right] \geq 0 & \forall \mathbb{P} \in \mathcal{P} & \Leftrightarrow \\
E_{\mathbb{P}}\left[\mathbb{1}_{F\left(\omega^{\prime}\right)} X\right] \geq 0 & \forall \mathbb{P} \in \mathcal{P} \text { s.t. } \mathbb{P}\left(\omega^{\prime}\right)>0 & \Leftrightarrow \\
E_{\mathbb{P}}\left[\mathbb{1}_{F\left(\omega^{\prime}\right)} X\right] / \mathbb{P}\left(\omega^{\prime}\right) \geq 0 & \forall \mathbb{P} \in \mathcal{P} \text { s.t. } \mathbb{P}\left(\omega^{\prime}\right)>0 & \Leftrightarrow \\
E_{\mathbb{P}}\left[X \mid \omega^{\prime}\right] \geq 0 & \forall \mathbb{P} \in \mathcal{P} \text { s.t. } \mathbb{P}\left(\omega^{\prime}\right)>0 & \Leftrightarrow \\
\phi\left(X \mid \omega^{\prime}\right) \geq 0 . & &
\end{aligned}
$$


Conversely, assume that $\phi$ is conditionally consistent and that $\phi(\cdot \mid 0)$ is represented by $\mathcal{P}$ as in (2.1). From the definition of conditional consistency it immediately follows that in (6.1) we can indeed take $\mathcal{P}_{\omega^{\prime}}=\mathcal{P}\left(\omega^{\prime}\right)$, cf. (6.6).

Claim 2.

First assume that $\phi$ is representable, via (6.9), by a juncted test set $\mathcal{P}$. We verify Condition 3 of Theorem 4.2. From Claim 1 it follows that $\phi$ is conditionally consistent, so Lemma 4.4 is applicable, and hence

$$
\phi\left(X \mid \omega^{\prime} \alpha\right)=0 \text { for all } \alpha \in A \Rightarrow \phi\left(X \mid \omega^{\prime}\right) \geq 0 .
$$

Next we prove the implication with the reversed inequality. Let $\epsilon>0$ be given. By assumption, $\phi\left(X \mid \omega^{\prime} \alpha\right)=0$ for all $\alpha \in A$, so for each $\alpha \in A$, there is a test measure $\mathbb{P}^{\alpha} \in \mathcal{P}\left(\omega^{\prime} \alpha\right)$ such that $E_{\mathbb{P} \alpha} X<\epsilon$. By the junctedness assumption, there is a test measure $\mathbb{P} \in \overline{\mathcal{P}\left(\omega^{\prime}\right)}$ such that $E_{\mathbb{P}} X \in \operatorname{ch}\left\{E_{\mathbb{P}_{\alpha}} X\right\}$, so that $E_{\mathbb{P}} X<\epsilon$. It follows that $\inf _{\mathbb{P} \in \mathcal{P}\left(\omega^{\prime}\right)} E_{\mathbb{P}} X \leq 0$. Together with (7.1) this implies Condition 3 of Theorem 4.2, so the characterization in Claim 2 is sufficient for sequential consistency.

Conversely, let $\phi$ be coherent, conditionally consistent, and sequentially consistent. It follows from Claim 1 that $\phi$ is globally representable by a test set $\mathcal{P}$ which we may assume to be convex, by Lemma A.2 in the Appendix. Suppose that $\mathcal{P}$ is not juncted. By definition, this means that there is a node $\omega^{\prime} \in \Omega^{\prime \prime}$ and a corresponding selection $\left\{\mathbb{P}_{\alpha}\right\}_{\alpha \in A}$, with $\mathbb{P}_{\alpha} \in \overline{\mathcal{P}\left(\omega^{\prime} \alpha\right)}$, such that this selction admits no junct in $\overline{\mathcal{P}\left(\omega^{\prime}\right)}$. Let $\mathcal{C}$ denote the set of all juncts of $\left\{\mathbb{P}_{\alpha}\right\}_{\alpha \in A}$ in $\operatorname{Pr}\left(\omega^{\prime}\right)$. The set $\mathcal{C}$ is closed and convex, and the same holds for $\overline{\mathcal{P}\left(\omega^{\prime}\right)}$. Consequently, there is a hyperplane strictly separating $\mathcal{P}^{\prime}$ and $\mathcal{C}$. Since the acceptability measure associated to $\overline{\mathcal{P}\left(\omega^{\prime}\right)}$ is $\phi\left(\cdot \mid \omega^{\prime}\right)$, this means that there is an $X \in \mathcal{X}(\Omega)$ with $\phi\left(X \mid \omega^{\prime}\right)>0$ and $E_{\mathbb{P}} X<0$ for all $\mathbb{P} \in \mathcal{C}$. The set $\mathcal{C}$ contains in particular all tests $\mathbb{P}$ with $\mathbb{P}(\alpha)=1$ and $\mathbb{P}(\cdot \mid \alpha)=\mathbb{P}_{\alpha}$, so the latter inequality implies that $\phi\left(X \mid \omega^{\prime} \alpha\right)<0$ for all $\alpha \in A$. This means that Condition 1 in Theorem 4.2 does not hold, and hence $\phi$ is not sequentially consistent. In this way, the "only if" part of Claim 2 is proved.

Claim 3.

In view of Thm.4.1, the claim is immediate from the fact that dynamic consistency is characterized by the product property (see for instance Roorda et al. (2004), Theorem $2.12)$.

The proof of Claim 2 shows that any convex global test set of a sequentially consistent $\phi$ must be juncted, and hence that junctedness is preserved under taking the convex hull of a test set. Another conclusion that may be drawn is that the stability property introduced in Artzner et al. (2004) is, at least in the case of a finite scenario set, equivalent to the product property; this follows because the tower law property is taken as the definition of 
time consistency in the cited paper and proved there to be characterized by the stability property.

\section{Multistep Tail Value at Risk}

We apply our results to develop multiperiod versions of Tail Value at Risk (TVaR). In this context we need a reference measure. Throughout this section we assume, as an extension of the standard framework described in Subsection 2.2, that a fixed reference measure $\mathbb{P}^{*}$ on $\Omega$ is given, and that $\mathbb{P}^{*}(\omega)>0$ for all $\omega \in \Omega$.

Our starting point is the definition in Artzner et al. (2004), which applies to settings in which $\Omega$ is not necessarily finite. For a given reference measure $\mathbb{P}^{*}$, Artzner et al. define the single-period TVaR of level $\lambda \in(0,1]$ by

$$
\begin{aligned}
\operatorname{TVaR}_{\lambda}(X) & =\inf _{\mathbb{P}}\left\{E_{\mathbb{P}}[X] \mid \frac{d \mathbb{P}}{d \mathbb{P}^{*}} \leq \lambda^{-1}\right\} \\
& =\inf _{Z}\left\{E_{\mathbb{P}^{*}}[Z X] \mid 0 \leq Z \leq \lambda^{-1}, E_{\mathbb{P}^{*}}[Z]=1\right\}
\end{aligned}
$$

where $\frac{d \mathbb{P}}{d \mathbb{P}^{*}}$ denotes the Radon-Nikodým $(\mathrm{RN})$ derivative of $\mathbb{P}$ with respect to $\mathbb{P}^{*}$, and $Z X$ is the position $\omega \mapsto Z(\omega) X(\omega)$. As synonyms or slightly different variants, also the terms Expected Shortfall, Conditional Value-at-Risk, Average Value-at-Risk, Tail Conditional Expectation, and Worst Conditional Expectation are used in the literature. Usage across different papers is not entirely systematic. In this paper, the term TVaR refers to a coherent risk measure, as is obvious from the expression (8.1) and the representation result Thm. 2.1.

In our setting with finite outcome space $\Omega$, the bound on the RN derivative can be written as a restriction on the probabilities of individual scenarios:

$$
\frac{d \mathbb{P}}{d \mathbb{P}^{*}} \leq \lambda^{-1} \Leftrightarrow \lambda \mathbb{P}(\omega) \leq \mathbb{P}^{*}(\omega) \text { for all } \omega \in \Omega
$$

We define the level of a given test measure as the inverse of the infinity norm of its RN derivative:

$$
\lambda_{\mathbb{P}}:=\left\|\frac{d \mathbb{P}}{d \mathbb{P}^{*}}\right\|_{\infty}^{-1}=\max _{\lambda \in[0,1]}\left\{\lambda \mid \lambda \mathbb{P}(\omega) \leq \mathbb{P}^{*}(\omega) \text { for all } \omega \in \Omega\right\}=\min _{\omega \in \Omega, \mathbb{P}(\omega)>0} \frac{\mathbb{P}^{*}(\omega)}{\mathbb{P}(\omega)} .
$$

Similarly we define for conditional measures

$$
\lambda_{\mathbb{P}\left(\cdot \mid \omega^{\prime}\right)}=\max _{\lambda \in[0,1]}\left\{\lambda \mid \lambda \mathbb{P}(\omega) \mathbb{P}^{*}\left(\omega^{\prime}\right) \leq \mathbb{P}\left(\omega^{\prime}\right) \mathbb{P}^{*}(\omega) \text { for all } \omega \in F\left(\omega^{\prime}\right)\right\} .
$$

This implies in particular that $\lambda_{\mathbb{P}\left(\cdot \mid \omega^{\prime}\right)}=1$ in case $\mathbb{P}\left(\omega^{\prime}\right)=0$.

A natural idea, also considered in Artzner et al. (2004) in a setting in which $\Omega$ is infinite, is to define a multi-period version by taking $\phi\left(\cdot \mid \omega^{\prime}\right)$ as the TVaR measure of level $\lambda \in(0,1]$, 
conditioned on $\omega^{\prime}$, over the remaining period:

$$
\operatorname{TVaR}_{\lambda}\left(X \mid \omega^{\prime}\right)=\inf _{\mathbb{P} \in \operatorname{Pr}}\left\{E_{\mathbb{P}}\left[X \mid \omega^{\prime}\right] \mid \lambda_{\mathbb{P}\left(\cdot \mid \omega^{\prime}\right)} \geq \lambda\right\}
$$

The measure obtained in this way is in general not even conditionally consistent. This fact is illustrated by the following example, taken from Artzner et al. (2004).

EXAMPLE 8.1 Let $\Omega=\{u u, u m, u d, d u, d m, d d\},{ }^{7}$ and let the reference measure assign equal probabilities to all paths. Define $\phi$ as the measure $\mathrm{TVaR}_{\lambda}$ with $\lambda=2 / 3$. In notation similar to previous examples, consider the position $G$ given by $G=[-10,12,14,-20,22,22]$. Computation shows that $\phi(G \mid 0)=-1$, while $\phi(G \mid u)=\phi(G \mid d)=1$. Since we know that under a conditionally consistent measure the evaluation at any node cannot be less than the minimum of the evaluations at all successor nodes (Lemma 4.4), it follows that $\phi$ is not conditionally consistent. Consequently, $\phi$ does not admit a representation in the form (6.9). Indeed, as observed in Artzner et al. (2004), different test sets are used in different states.

In order to avoid conditional inconsistency, one can consider the acceptability measure $\phi^{\prime}$ represented by the test set corresponding to $\mathrm{TVaR}_{\lambda}$ in (8.1) via (6.9). The resulting measure is conditionally consistent by construction, but not sequentially consistent. In fact, one finds that $\phi^{\prime}(G)=-1$ as before, but now $\phi^{\prime}(G \mid u)=-10$ and $\phi^{\prime}(G \mid d)=-20$. So the position $G+2$ is initially considered acceptable, while it is obviously unacceptable in the next period.

The example illustrates that conditional consistency alone is too weak to rule out consequences that would be considered undesirable in many applications. In this section we develop two alternative versions of TVaR that satisfy the stronger consistency conditions considered in this paper.

\subsection{Dynamically consistent TVaR}

With the test set characterization of Theorem 7.1 in mind, it is an obvious idea to define a dynamically consistent variant considering the product-type test set constructed from singleperiod test sets of TVaR type. If the same level $\lambda$ for TVaR is used at all nodes, the test set obtained in this way is

$$
\mathcal{P}_{\lambda}^{\times}:=\left\{\mathbb{P} \in \operatorname{Pr} \mid \lambda_{\mathbb{P}^{\mathrm{s}}\left(\cdot \mid \omega^{\prime}\right)} \geq \lambda \text { for all } \omega^{\prime} \in \Omega^{\prime \prime}\right\} .
$$

Definition 8.2 For a given reference measure $\mathbb{P}^{*}$ and a given real number $\lambda \in(0,1]$, the multiperiod acceptability measure $\phi_{\mathcal{P}_{\lambda}^{\times}}$is called dynamically consistent TVaR (DTVaR) of level $\lambda$.

\footnotetext{
${ }^{7}$ To bring this in line with our notation, take event set $A=\{u, m, d\}$, and let the event $m$ have zero probability in the first step.
} 
To facilitate comparison with the definition (8.5) of $\mathrm{TVaR}_{\lambda}$, we can also write

$$
\begin{array}{r}
\operatorname{DTVaR}_{\lambda}\left(X \mid \omega^{\prime}\right)=\inf _{\mathbb{P} \in \operatorname{Pr}}\left\{E_{\mathbb{P}}\left[X \mid \omega^{\prime \prime}\right] \mid \lambda_{\mathbb{P s}}\left(\cdot \mid \omega^{\prime \prime}\right) \geq \lambda \text { for all } \omega^{\prime \prime} \in F\left(\omega^{\prime}\right)\right\} \\
=\inf _{Z}\left\{E_{\mathbb{P}^{*}}[Z X] \mid 0 \leq E_{\mathbb{P}^{*}}\left[Z \mid \omega^{\prime \prime} \alpha\right] \leq \lambda^{-1} E_{\mathbb{P}^{*}}\left[Z \mid \omega^{\prime \prime}\right]\right. \\
\left.\forall \omega^{\prime \prime} \in F\left(\omega^{\prime}\right) \cap \Omega^{\prime \prime}, E_{\mathbb{P}^{*}}[Z]=1\right\} .
\end{array}
$$

The relevance condition (3.2) is clearly satisfied, since $\mathbb{P}^{*} \in \mathcal{P}_{\lambda}^{\times}$for all $\lambda$. It follows directly from Theorem 7.1.3 that $\mathrm{DTVaR}_{\lambda}$ satisfies the tower law. According to Theorem 4.1, it hence also satisfies the stepwise monotonicity condition, which clearly rules out the type of time inconsistency observed in Example 8.1, as illustrated below. In addition, a computational advantage of DTVaR is that it can be evaluated by dynamic programming, see Roorda et al. (2004).

EXAmPLE 8.3 Continuing the previous example, we now consider $\phi^{\times}:=\mathrm{DTVaR}_{\lambda}$, with $\lambda=\frac{2}{3}$ as before. Because the reference measure is uniform and we have two branches at the first step and three at the second step, the corresponding test set family is given by

$$
\mathcal{P}_{\lambda}^{\times}=\left\{\mathbb{P} \in \operatorname{Pr} \mid \lambda \mathbb{P}(\alpha) \leq \frac{1}{2}, \lambda \mathbb{P}\left(\alpha^{\prime} \mid \alpha\right) \leq \frac{1}{3} \text { for all } \alpha, \alpha^{\prime} \in A\right\}
$$

In the second step the evaluations are the same as the ones produced by the TVaR measure, so $\phi^{\times}(G \mid u)=\phi^{\times}(G \mid d)=1$. From the tower law (or Condition 1 in Theorem 4.1) it follows immediately that also $\phi^{\times}(G \mid 0)=1$, which demonstrates that the inconsistency in Example 8.1 has disappeared. Note that the combination of two single step tests with minimum level $\frac{2}{3}$ leads to an initial level of $\frac{4}{9}$.

\subsection{Sequentially consistent TVaR}

Next we introduce a sequentially consistent version of TVaR. The idea is to impose a bound not only on the initial level of a test, as in (8.5), but also on its tests conditional on future information. We define

$$
\begin{aligned}
& \mathcal{P}_{\lambda}=\left\{\mathbb{P} \in \operatorname{Pr} \mid \lambda_{\mathbb{P}\left(\cdot \mid \omega^{\prime}\right)} \geq \lambda \text { for all } \omega^{\prime} \in \Omega^{\prime \prime}\right\} \\
& =\left\{\mathbb{P} \in \operatorname{Pr} \mid \text { for all } \omega^{\prime} \in \Omega^{\prime \prime}, \omega \succeq \omega^{\prime}: \lambda \mathbb{P}(\omega) \mathbb{P}^{*}\left(\omega^{\prime}\right) \leq \mathbb{P}\left(\omega^{\prime}\right) \mathbb{P}^{*}(\omega)\right\} .
\end{aligned}
$$

Definition 8.4 For a given reference measure $\mathbb{P}^{*}$ and a given real number $\lambda \in(0,1]$, the multiperiod acceptability measure $\phi_{\mathcal{P}_{\lambda}}$ is called sequentially consistent TVaR (STVaR) of level $\lambda$. 
Correspondingly, we write

$$
\begin{aligned}
& \operatorname{STVaR}_{\lambda}\left(X \mid \omega^{\prime}\right)=\inf _{\mathbb{P} \in \operatorname{Pr}}\left\{E_{\mathbb{P}}\left[X \mid \omega^{\prime}\right] \mid \lambda_{\mathbb{P}\left(\cdot \mid \omega^{\prime \prime}\right)} \geq \lambda \text { for all } \omega^{\prime \prime} \in F\left(\omega^{\prime}\right)\right\} \\
& =\inf _{Z}\left\{E_{\mathbb{P}^{*}}[Z X] \mid E_{\mathbb{P}^{*}}[Z]=1 \text { and } 0 \leq Z(\omega) \leq \lambda^{-1} E_{\mathbb{P}^{*}}\left[Z|\omega|_{t}\right]\right. \\
& \text { for all } \left.t \text { and all } \omega \succeq \omega^{\prime}\right\} \text {. }
\end{aligned}
$$

Under our standing assumption that the reference measure $\mathbb{P}^{*}$ assigns positive weight to all trajectories $\omega \in \Omega$, the relevance axiom is always satisfied, since $\mathbb{P}^{*} \in \mathcal{P}_{\lambda}$ for all $\lambda$. To see that $\mathrm{STVaR}_{\lambda}$ is indeed sequentially consistent, we first state a relation between the level of a conditional measure at a given node and the levels of the conditional measures at successor nodes.

Lemma 8.5 Let $\left\{\mathbb{P}_{\alpha}\right\}_{\alpha \in A}\left(\mathbb{P}_{\alpha} \in \operatorname{Pr}\left(\omega^{\prime} \alpha\right)\right)$ be a collection of conditional measures at the successor nodes of a given node $\omega^{\prime} \in \Omega^{\prime \prime}$. Let $\mathbb{P}$ be a junct of this collection in $\operatorname{Pr}\left(\omega^{\prime}\right)$. The level of $\mathbb{P}$ is given by

$$
\lambda_{\mathbb{P}}=\min _{\alpha \in A, p_{\alpha}>0} \frac{p_{\alpha}^{*}}{p_{\alpha}} \lambda_{\alpha}
$$

where $\lambda_{\alpha}:=\lambda_{\mathbb{P}_{\alpha}}, p_{\alpha}:=\mathbb{P}^{\mathrm{s}}(\alpha)$, and $p_{\alpha}^{*}:=\mathbb{P}^{* \mathrm{~s}}(\alpha)$. Among the set of all juncts of the collection $\left\{\mathbb{P}_{\alpha}\right\}_{\alpha \in A}$, the one whose level is maximal is obtained by taking

$$
\mathbb{P}^{\mathrm{s}}(\alpha)=\frac{p_{\alpha}^{*} \lambda_{\alpha}}{\Sigma_{\alpha \in A} p_{\alpha}^{*} \lambda_{\alpha}} \quad(\alpha \in A)
$$

The corresponding maximal level is $\lambda^{\max }=\Sigma_{\alpha \in A} p_{\alpha}^{*} \lambda_{\alpha}$.

Proof By definition (8.3), we have

$$
\begin{aligned}
\lambda_{\mathbb{P}} & =\max _{\lambda}\left\{\lambda \mid \lambda \mathbb{P}(\omega) \leq \mathbb{P}^{*}(\omega) \text { for all } \omega \in F\left(\omega^{\prime}\right)\right\} \\
& =\max _{\lambda}\left\{\lambda \mid \text { for all } \alpha \in A: \lambda \mathbb{P}(\alpha) \mathbb{P}(\omega \mid \alpha) \leq \mathbb{P}^{*}(\alpha) \mathbb{P}^{*}(\omega \mid \alpha) \text { for all } \omega \in F\left(\omega^{\prime} \alpha\right)\right\} \\
& =\min _{\alpha \in A} \max _{\lambda}\left\{\lambda \mid \lambda \mathbb{P}(\alpha) \mathbb{P}(\omega \mid \alpha) \leq \mathbb{P}^{*}(\alpha) \mathbb{P}^{*}(\omega \mid \alpha) \text { for all } \omega \in F\left(\omega^{\prime} \alpha\right)\right\} \\
& =\min _{\alpha \in A, \mathbb{P}(\alpha)>0} \frac{\mathbb{P}^{*}(\alpha)}{\mathbb{P}(\alpha)} \max _{\lambda}\left\{\lambda \mid \lambda \mathbb{P}(\omega \mid \alpha) \leq \mathbb{P}^{*}(\omega \mid \alpha) \text { for all } \omega \in F\left(\omega^{\prime} \alpha\right)\right\} \\
& =\min _{\alpha \in A, p_{\alpha}>0} \lambda_{\alpha} p_{\alpha}^{*} / p_{\alpha} .
\end{aligned}
$$

Maximizing $\lambda_{\mathbb{P}}$ over $p_{\alpha}$ subject to the constraint $\sum_{\alpha} p_{\alpha}=1$ is a standard optimization problem; the maximum is obtained by taking the $p_{\alpha}$ 's proportional to $p_{\alpha}^{*} \lambda_{\alpha}$, which gives rise to the result stated in the theorem.

On the basis of the lemma, sequential consistency of STVaR can be proved easily.

Proposition 8.6 The multiperiod acceptability measure $\operatorname{STVaR}_{\lambda}(\lambda \in(0,1])$ is sequentially consistent. 
Proof In view of Thm. 4.2, what we have to show is that the global test set $\mathcal{P}_{\lambda}$ is juncted. Take $\omega^{\prime} \in \Omega^{\prime \prime}$, and let $\left\{\mathbb{P}_{\alpha}\right\}_{\alpha \in A}$ be a selection of conditional tests with $\mathbb{P}_{\alpha} \in \mathcal{P}_{\lambda}\left(\omega^{\prime} \alpha\right)$, so that $\lambda_{\alpha}:=\lambda_{\mathbb{P}_{\alpha}} \geq \lambda$ for all $\alpha \in A$. According to the lemma, the maximal level of juncts of the given selection is $\sum_{\alpha \in A} p_{\alpha}^{*} \lambda_{\alpha}$, and since $\lambda_{\alpha} \geq \lambda$ for all $\alpha$ and $\sum p_{\alpha}^{*}=1$ this maximum is equal to or larger than $\lambda$. In other words, there is a junct in $\mathcal{P}_{\lambda}\left(\omega^{\prime}\right)$. In fact, the lemma shows that it suffices to construct a junct using the single-step probabilities of the reference measure at $\omega^{\prime}$.

Obviously $\mathrm{STVaR}_{\lambda} \geq \mathrm{TVaR}_{\lambda}$, and $\mathrm{STVaR}_{\lambda}$ is in fact the smallest conditionally consistent multiperiod acceptability measure not involving conditional test levels below $\lambda$. This follows directly from the fact that $\mathcal{P}_{\lambda}$ is the largest test set with the property that $\mathcal{P}_{\lambda}\left(\omega^{\prime}\right)$ is contained in the test set corresponding to $\operatorname{TVaR}_{\lambda}\left(\cdot \mid \omega^{\prime}\right)$ for every $\omega^{\prime} \in \Omega^{\prime}$.

From the computational point of view, it should be noted that STVaR is not necessarily dynamically consistent, and hence cannot be evaluated by Dynamic Programming in general. However, the test set $\mathcal{P}_{\lambda}$ is a polytope, as is seen from the second expression in (8.9), so STVaR can be computed by linear programming methods.

Example 8.7 Consider the setting of Example 8.1 again. Let $\phi^{+}$denote the acceptability measure $\operatorname{STVaR}_{\lambda}$ with $\lambda=\frac{2}{3}$. As noted above, the associated test set $\mathcal{P}_{2 / 3}$ is a polytope. It can be shown, for instance by an application of the Avis-Fukuda algorithm, ${ }^{8}$ that $\mathcal{P}_{2 / 3}$ has in fact 15 vertices, which may be described as follows. Nine vertices are characterized by $\mathbb{P}(u)=\mathbb{P}(d)=1 / 2$ and by equal weights placed on two out of the three successor states for each of the two possible states at time 1 . In three tests, $\mathbb{P}(u)=3 / 4, \mathbb{P}(\cdot \mid u)$ is uniform, $\mathbb{P}(d)=1 / 4$, and $\mathbb{P}(\cdot \mid d)$ puts equal weights on two out of the three successor states to $d$. The remaining three vertices are of the same type with the roles of $u$ and $d$ interchanged. It follows that $\phi^{+}(G \mid u)=\phi^{+}(G \mid d)=\phi^{+}(G \mid 0)=1$. As in the previous example, TVaR's time consistency problem has been eliminated.

\subsection{Is DTVaR better than STVaR?}

The acceptability measure DTVaR satisfies a stronger notion of time consistency than STVaR does. The question may be posed whether this means that DTVaR should be preferred to STVaR as a basis for taking decisions under uncertainty. More generally, one may ask in which situations dynamic consistency is an appropriate requirement to impose.

In the context of individual decision making, examples have been given by Wu (1999) and Epstein and Schneider (2003) to show that dynamic consistency may well be violated

\footnotetext{
${ }^{8}$ See http://library.wolfram.com/infocenter/MathSource/440/ for an implementation of this algorithm in Mathematica.
} 
by agents who are subject to ambiguity aversion or anxiety. Eichberger et al. (2005, p. 150) state that there is in fact very little experimental evidence that human decision making is dynamically consistent. But even if dynamic consistency may fail for descriptive purposes, the axiom may still be important in a normative context.

There are various normative uses of acceptability measures, such as derivative pricing, insurance premium setting, and regulatory applications. Of course, the appropriateness of imposing dynamic consistency may depend on the specific application at hand. For instance, in a regulatory context an acceptability measure may be used to determine the amount of economic capital that is needed in connection with a given position. If a coherent risk measure is used, the capital charge is viewed as a certainty equivalent; for instance, if the charge for a certain risky position is 100, then the position is equivalent (from the point of view of the acceptability measure) to a sure loss of 100 . One may ask, however, whether these positions should still be considered equivalent when looked at from an earlier point in time. The presence of a small chance of arriving at a situation in which a capital reservation of 100 has to be made may not have the same impact as the presence of an equally small chance of incurring a loss of 100. This point is worked out more quantitatively in the following example.

Example 8.8 Consider a two-step model with $A=\{u, d\}$. The reference measure $\mathbb{P}^{*}$ is defined by $\mathbb{P}^{*}(d)=0.01$ and $\mathbb{P}^{*}(d \mid \alpha)=0.01$ for $\alpha \in A$. We evaluate positions by DTVaR and by STVaR, both at level $\lambda=0.05$. First consider a position $X$ that leads to a loss of 20 if $d u$ or $d d$ occurs, and that has zero payoff in the other two cases. The two acceptability measures completely agree in their evaluation of this position, which is no surprise since we essentially have a single-period problem. Both measures generate evaluations -20 and 0 at nodes $d$ and $u$ respectively, and they evaluate the position $X$ to -4 at time 0 . Now consider a position $Y$ that generates a loss of 100 if the event $d d$ occurs and that has zero payoff in other cases. With respect to this position, DTVaR and STVaR still agree at nodes $u$ and $d$; moreover, their evaluations at these nodes are the same for position $Y$ as they are for position $X$. The dynamic consistency of DTVaR then implies that the evaluation of $Y$ at time 0 under DTVaR is also the same as the evaluation of $X$, namely -4 . In contrast, we have $\operatorname{STVaR}_{0.05}(Y)=-0.2$.

One may debate which one of the evaluations of the position $Y$ at time 0 is more appropriate. Note that $Y$ represents (according to the reference measure) a $0.01 \%$ probability of a loss of 100 , whereas $X$ represents a $1 \%$ probability of a loss of 20 . The two positions lead to equal capital charges under DTVaR, whereas STVaR places a much lighter charge on the first position. Due to the low value of $\lambda$, the DTVaR measure is subject to a strong multiplicative effect which is unavoidable if this value of $\lambda$ is considered appropriate for 
single-period risk aversion at all nodes.

In the example, we used a rather low value of the parameter $\lambda$, which puts great emphasis on negative outcomes. Such a low value of $\lambda$ may be justifiable in a regulatory context. If DTVaR would be used for pricing, then it is more likely that a value of $\lambda$ close to 1 would be chosen, so as to give more weight to upward potential relative to downward risk. The multiplicative effect that was mentioned in the example would then not be felt as strongly. It appears that the effect of TailVaR-related measures can be varied considerably by changing the level parameter $\lambda$.

\section{Conclusions}

It has been the purpose of this paper to discuss a number of different notions of time consistency for multiperiod acceptability measures, and, in the case in which we consider coherent measures, to provide characterizations of these notions in terms of families of test measures. Under a nondegeneracy assumption expressing that each scenario has some impact on acceptability, and assuming that we consider coherent measures, our results can be summarized as follows. One notion of time consistency is what we call conditional consistency; it expresses that a position that has a payoff only when a certain event occurs is acceptable initially if and only if it is acceptable in the situation when this event actually does take place. This condition is weaker than a second notion called sequential consistency. Under the latter notion of consistency, it cannot happen that a position would be considered acceptable in a given state whereas it is unacceptable in each of its successor states, or vice versa. Sequential consistency in turn is weaker than dynamic consistency which means that positions cannot be evaluated differently in a given state when their evaluations in each of the successor states are the same. Finally, dynamic consistency is equivalent to the tower law of conditional evaluations, which requires that the evaluation of a position is not changed when its payoffs on scenarios following a certain event are replaced by the evaluation of the position conditional on that event.

From the basic representation theorem for coherent acceptability measures, it follows that any coherent multiperiod acceptability measure can be represented by a family of collections of restricted probability measures. Extending earlier results concerning the representation of dynamically consistent measures, we have established which special properties should be satisfied by the representing family so that the corresponding acceptability measures should be time consistent in weaker senses. Conditional consistency, the weakest notion of time consistency, holds if and only if the representing family of collections of probability measures is derived by conditioning from a given collection of probability measures on the complete 
scenario set. This means that specification of conditionally consistent coherent acceptability measures is much simpler than specification of coherent multiperiod acceptability measures in general; instead of specifying collections of probability measures on future scenarios at each separate node, it suffices to specify just one collection. In the case of a dynamically consistent acceptability measure, a further restriction is imposed in that the collection of overall probability measures should be constructed as a product of single-step probability measures. This characterization is already known from earlier work. The product property implies a certain relation between the collection of induced measures at a given node and the collections at its successor nodes, which is not in general satisfied by test set families obtained by conditioning from a global test set. This relation, which we have called "junctedness", has been shown in this paper to characterize sequential consistency.

As an application, we have considered various dynamic extensions of the well known TailValue-at-Risk measure. One such extension was already discussed in Artzner et al. 2004 and was shown in that paper not to be dynamically consistent. Here it has been shown that this dynamic version of TVaR is actually not even conditionally consistent. Other dynamic variants of TVaR can be considered, however, and we have discussed several possibilities that satisfy stronger consistency axioms.

Several questions suggest themselves as directions for further research. For many applications in finance, it would be convenient to work with continuous-time and continuous-state versions of acceptability measures. In the continuous-time continuous-state context, the axiomatic approach is much more challenging than in the finite framework that we have used in this paper. Nevertheless, the results that have been obtained here may to some extent provide hints for the specification of continuous-time measures, even when a rigorous axiomatic framework is lacking. The appropriateness of a given risk measure of course depends on the intended application (such as regulation, pricing in incomplete markets, or insurance premium setting), and depends also on whether one takes a normative or a descriptive point of view; this motivates more study of concrete dynamic acceptability measures and their properties. In this paper we have mainly considered coherent risk measures; it may be expected that most of the results have generalizations for convex acceptability measures as discussed for instance in Föllmer and Schied (2004). Similarly, we have not considered acceptability under hedging, but extension of our results to this case would seem promising (cf. again Föllmer and Schied (2004), Staum (2004), and Roorda et al. (2004)). The computation of hedge policies that are optimal with respect to a given acceptability measure is a major research issue; for instance, optimization in a multiperiod framework but with respect to an atemporal notion of TVaR is discussed in Rockafellar and Uryasev (2002). 


\section{A Appendix}

The following lemma will be used in the proof of Thm.4.1.

Lemma A.1 Let $\phi$ be a dynamically consistent acceptability measure, and let $X$ and $Y$ be given positions. Suppose there exists a stopping time $\tau$ such that

$$
\phi\left(X \mid \omega^{\prime}\right)=\phi\left(Y \mid \omega^{\prime}\right) \quad \text { for all } \omega^{\prime} \in \Omega_{\tau} .
$$

Under these conditions, we have $\phi(X)=\phi(Y)$.

Proof If $\tau=0$, the conclusion is immediate, since $\Omega_{0}=\{0\}$. Suppose now that $\tau \neq 0$. Define a sequence $\tau_{0}, \tau_{1}, \ldots$ of stopping times recursively by

$$
\begin{aligned}
\tau_{0} & =\tau \\
\tau_{k+1} & =\tau_{k} \wedge\left[0 \vee\left(\left(\max \tau_{k}\right)-1\right)\right]
\end{aligned}
$$

where, of course, $\max \tau_{k}=\max _{\omega} \tau_{k}(\omega)$. A simple inductive argument shows that each $\tau_{k}$ is indeed a stopping time, using the fact that the minimum of two stopping times (and, in particular, the minimum of a deterministic time and a stopping time) is again a stopping time. Since $\max \tau_{k+1}=\left(\max \tau_{k}\right)-1$ for all $k$ such that $\max \tau_{k} \geq 1$, it is clear that the sequence above reaches 0 in finitely many steps. Therefore, it is sufficient to show that, if $\phi\left(X \mid \omega^{\prime}\right)=\phi\left(Y \mid \omega^{\prime}\right)$ for all $\omega^{\prime} \in \Omega_{\tau_{k}}$, then the statement also holds for all $\omega^{\prime} \in \Omega_{\tau_{k+1}}$.

To prove this claim, take $\omega^{\prime} \in \Omega_{\tau_{k+1}}$. If $\omega^{\prime} \in \Omega_{\tau_{k}}$, then the conclusion already follows from (A.1); so assume that $\omega^{\prime} \notin \Omega_{\tau_{k}}$. Take $\omega \in F\left(\omega^{\prime}\right)$. We cannot have $\tau_{k}(\omega)=T\left(\omega^{\prime}\right)$ because that would imply that $\omega^{\prime} \in \Omega_{\tau_{k}}$, but on the other hand we must have $\tau_{k}(\omega) \leq T\left(\omega^{\prime}\right)+1$ by the definition of $\tau_{k+1}$. Therefore, $\tau_{k}(\omega)=T\left(\omega^{\prime}\right)+1$. From this it follows that $\left.\omega\right|_{T\left(\omega^{\prime}\right)+1} \in \Omega_{\tau_{k}}$. Since this reasoning applies to all $\omega \in F\left(\omega^{\prime}\right)$, it follows that $\omega^{\prime} \alpha \in \Omega_{\tau_{k}}$ for all $\alpha \in A$. Using now the assumption that $\phi$ is dynamically consistent, we obtain the desired conclusion that $\phi\left(X \mid \omega^{\prime}\right)=\phi\left(Y \mid \omega^{\prime}\right)$. This completes the proof.

\section{Proof of Theorem 4.1}

Condition $1 \Rightarrow$ Condition 2.

Condition 1 implies, together with the soundness property, that the certainty equivalent of a given position at a given node is determined in terms of its certainty equivalents at all successor nodes. So if $\phi\left(X \mid \omega^{\prime} \alpha\right)=\phi\left(Y \mid \omega^{\prime} \alpha\right)$ for all $\alpha$, then $\phi\left(X \mid \omega^{\prime}\right)=\phi\left(Y \mid \omega^{\prime}\right)$; in other words, Condition 2 holds.

Condition $2 \Rightarrow$ Condition 1 .

Assume that $\phi$ is dynamically consistent. Take $X \in \mathcal{X}(\Omega)$ and $\omega^{\prime} \in \Omega^{\prime \prime}$; define $Y:=$ $\mathcal{E}_{T\left(\omega^{\prime}\right)+1}^{\phi} X$. We have to prove that $\phi\left(X \mid \omega^{\prime}\right)=\phi\left(Y \mid \omega^{\prime}\right)$. Since $\phi$ is dynamically consistent, 
it suffices to show that $\phi\left(X \mid \omega^{\prime} \alpha\right)=\phi\left(Y \mid \omega^{\prime} \alpha\right)$ for all $\alpha \in A$. By definition of $Y$, we have indeed, for any $\alpha \in A, \phi\left(Y \mid \omega^{\prime} \alpha\right)=\phi\left(\phi\left(X \mid \omega^{\prime} \alpha\right) \mid \omega^{\prime} \alpha\right)=\phi\left(X \mid \omega^{\prime} \alpha\right)$.

Necessity of Condition 1.

This is obvious.

\section{Sufficiency of Condition 1.}

Assume that $\phi$ satisfies Condition 1 . Take $X \in \mathcal{X}(\Omega)$ and $\omega^{\prime} \in \Omega^{\prime}$. Without loss of generality, we can take $\omega^{\prime}=0$; for other $\omega^{\prime} \in \Omega^{\prime}$, the proof is essentially the same (use $F\left(\omega^{\prime}\right)$ instead of $\Omega)$. Let $\tau$ be a stopping time. We have to prove that $\phi\left(\mathcal{E}_{\tau}^{\phi} X\right)=\phi(X)$. Define a sequence of stopping times $\left(\tau_{0}, \tau_{1}, \ldots\right)$ as in (A.2). Since $\phi\left(\mathcal{E}_{0}^{\phi} X\right)=\phi((\phi(X)))=\phi(X)$, it is sufficient to show that $\phi\left(\mathcal{E}_{\tau_{k+1}}^{\phi} X\right)=\phi\left(\mathcal{E}_{\tau_{k}}^{\phi} X\right)$ for all $k$. By Lemma A.1, this follows if

$$
\phi\left(\mathcal{E}_{\tau_{k+1}}^{\phi} X \mid \omega^{\prime}\right)=\phi\left(\mathcal{E}_{\tau_{k}}^{\phi} X \mid \omega^{\prime}\right) \quad \text { for all } \omega^{\prime} \in \Omega_{\tau_{k+1}}
$$

This claim holds true by Condition 1 .

Equivalence of Conditions 2 and 3.

This has been proved in Roorda et al. (2004), Proposition 2.6.

\section{Proof of theorem 4.2}

Necessity and sufficiency of Condition 1.

Sequential consistency clearly implies Condition 1. Conversely, whenever $\phi\left(X \mid \omega^{\prime}\right) \geq 0$, a full path $\omega \in F\left(\omega^{\prime}\right)$ can be constructed for which $X$ remains acceptable, by exploiting Condition 1 repeatedly. A similar result holds for the reversed inequality, and it follows that $\phi$ must be sequentially consistent.

Equivalence of Conditions 1 and 2.

Condition 2 clearly implies Condition 1. Conversely, assume Condition 2 does not hold. Then there exist $\omega^{\prime} \in \Omega^{\prime \prime}, X \in \mathcal{X}(\Omega)$, for which $\phi\left(X \mid \omega^{\prime}\right)$ outside the interval $\left[c_{\min }, c_{\max }\right]$, with $c_{\min }:=\min _{\alpha \in A} \phi\left(X \mid \omega^{\prime} \alpha\right)$ and $c_{\max }:=\max _{\alpha \in A} \phi\left(X \mid \omega^{\prime} \alpha\right)$. Clearly then $X-\phi\left(X \mid \omega^{\prime}\right)$ violates Condition 1.

Equivalence of Conditions 2 and 3.

Condition 3 is clearly implied by Condition 2. To prove the opposite implication, assume Condition 3 holds. Consider for any position $X \in \mathcal{X}(\Omega)$ and any $\omega^{\prime} \in \Omega^{\prime \prime}$, the position $X^{\prime}:=X-Y$ with $Y:=\mathcal{E}_{T\left(\omega^{\prime}\right)+1}^{\phi}(X)=\Sigma_{\alpha \in A} \mathbb{1}_{F\left(\omega^{\prime} \alpha\right)} \phi\left(X \mid \omega^{\prime} \alpha\right)$. Then $\phi\left(X^{\prime} \mid \omega^{\prime} \alpha\right)=0$ for all $\alpha \in A$, and Condition 3 hence implies that $\phi\left(X^{\prime} \mid \omega^{\prime}\right)=0$. With $c_{\min }, c_{\max }$ defined as before, clearly $c_{\min } \mathbb{1}_{F\left(\omega^{\prime}\right)} \leq Y \leq c_{\max } \mathbb{1}_{F\left(\omega^{\prime}\right)}$, so $X^{\prime}+c_{\min } \mathbb{1}_{F\left(\omega^{\prime}\right)} \leq X \leq X^{\prime}+c_{\max } \mathbb{1}_{F\left(\omega^{\prime}\right)}$. Translation invariance and monotonicity of $\phi\left(\cdot \mid \omega^{\prime}\right)$ now imply that $c_{\min } \leq \phi\left(X \mid \omega^{\prime}\right) \leq c_{\max }$, hence Condition 2 holds. 
Condition $4 \Rightarrow$ Condition 3.

This implication is easily established by using (3.1) twice, once with $X=0$ and once with $Y=0$.

Condition $2 \Rightarrow$ Condition 4 .

This is trivial.

\section{Proof of Theorem 4.3}

Necessity of Condition 1.

Assume $\phi$ is conditionally consistent. We have to prove that that for all $\omega^{\prime} \in \Omega^{\prime \prime}, \alpha \in A$,

$$
\phi\left(X \mid \omega^{\prime} \alpha\right) \geq 0 \Leftrightarrow \phi\left(\mathbb{1}_{F\left(\omega^{\prime} \alpha\right)} X \mid \omega^{\prime}\right) \geq 0
$$

Applying the conditional consistency property in Definition 3.1 shows that the left-hand side is equivalent to the condition $\phi\left(\mathbb{1}_{F\left(\omega^{\prime} \alpha\right)} X\right) \geq 0$, and that the right-hand side is equivalent to $\phi\left(\mathbb{1}_{F\left(\omega^{\prime}\right)} \mathbb{1}_{F\left(\omega^{\prime} \alpha\right)} X\right) \geq 0$. The equivalence (A.4) is now a direct consequence.

\section{Sufficiency of Condition 1.}

We prove that Condition 1 cannot hold if $\phi$ is not conditionally consistent. If $\phi$ is conditionally inconsistent, there exists an $\omega^{\prime} \in \Omega^{\prime}, X \in \mathcal{X}(\Omega)$, for which either (i) $\phi\left(\mathbb{1}_{F\left(\omega^{\prime}\right)} X\right) \geq 0$ and $\phi\left(X \mid \omega^{\prime}\right)<0$, or (ii) $\phi\left(\mathbb{1}_{F\left(\omega^{\prime}\right)} X\right)<0$ and $\phi\left(X \mid \omega^{\prime}\right) \geq 0$. Assume case (i); case (ii) is similar. Define $X^{\prime}:=\mathbb{1}_{F\left(\omega^{\prime}\right)} X$, and consider the sequence $\left\{\phi\left(X^{\prime}\left|\omega^{\prime}\right|_{t}\right)\right\}_{t=0, \ldots, T\left(\omega^{\prime}\right)}$. This sequence must contain at least one transition from nonnegative to negative, say from $\omega^{\prime \prime}$ to $\omega^{\prime \prime} \alpha \preceq \omega^{\prime}$. Then $\phi\left(X^{\prime} \mid \omega^{\prime \prime}\right)=\phi\left(\mathbb{1}_{F\left(\omega^{\prime \prime} \alpha\right)} X^{\prime} \mid \omega^{\prime \prime}\right) \geq 0$ and $\phi\left(X^{\prime} \mid \omega^{\prime \prime} \alpha\right)<0$, contradicting Condition 1.

Equivalence of Conditions 1 and 2.

Condition 2 clearly implies Condition 1. Also, the second part of Condition 2 is immediate from Condition 1. We now prove the first part of Condition 2 from Condition 1. Assume first that $\phi\left(X \mid \omega^{\prime} \alpha\right) \geq 0$. By Condition 1, we then have $\phi\left(\mathbb{1}_{F\left(\omega^{\prime} \alpha\right)} X \mid \omega^{\prime}\right) \geq 0$. Let $\epsilon>0$ be given, and define $\eta=\phi\left(X \mid \omega^{\prime} \alpha\right)+\epsilon$. We want to show that $\phi\left(\mathbb{1}_{F\left(\omega^{\prime} \alpha\right)} X \mid \omega^{\prime}\right) \leq \eta$. From $\phi\left(X \mid \omega^{\prime} \alpha\right)<\eta$ we have $\phi\left(X-\eta \mid \omega^{\prime} \alpha\right)<0$, so that, by Condition $1, \phi\left(\mathbb{1}_{F\left(\omega^{\prime} \alpha\right)}(X-\right.$ $\left.\eta) \mid \omega^{\prime}\right)<0$. By monotonicity and the translation property, and because $\eta \geq 0$, we have $-\phi\left(-\mathbb{1}_{F\left(\omega^{\prime} \alpha\right)} \eta \mid \omega^{\prime}\right) \leq \eta$. Therefore we can write, using superadditivity,

$$
\phi\left(\mathbb{1}_{F\left(\omega^{\prime} \alpha\right)} X \mid \omega^{\prime}\right) \leq \phi\left(\mathbb{1}_{F\left(\omega^{\prime} \alpha\right)}(X-\eta) \mid \omega^{\prime}\right)-\phi\left(-\mathbb{1}_{F\left(\omega^{\prime} \alpha\right)} \eta \mid \omega^{\prime}\right)<\eta .
$$

Similarly, it can be shown that $\phi\left(X \mid \omega^{\prime} \alpha\right)<0$ implies $0>\phi\left(\mathbb{1}_{F\left(\omega^{\prime} \alpha\right)} X \mid \omega^{\prime}\right) \geq \phi\left(X \mid \omega^{\prime} \alpha\right)$.

\section{Additional claim}

Assume that $\phi$ is strongly relevant and that Condition 1 holds. Let $X, \omega^{\prime}$, and $\alpha$ be such that $\phi\left(\mathbb{1}_{F\left(\omega^{\prime} \alpha\right)} X \mid \omega^{\prime}\right)=0$, and write $\eta:=\phi\left(X \mid \omega^{\prime} \alpha\right)$. Condition 1 implies that $\eta \geq 0$. By 
the translation property, we have $\phi\left(X-\eta \mid \omega^{\prime} \alpha\right)=0$. Using Condition 1 again, we find that $\phi\left(\mathbb{1}_{F\left(\omega^{\prime} \alpha\right)}(X-\eta) \mid \omega^{\prime}\right) \geq 0$. On the other hand, we also have $\phi\left(\mathbb{1}_{F\left(\omega^{\prime} \alpha\right)}(X-\eta) \mid \omega^{\prime}\right) \leq$ $\phi\left(\mathbb{1}_{F\left(\omega^{\prime} \alpha\right)} X \mid \omega^{\prime}\right)=0$. It follows that

$$
\phi\left(\mathbb{1}_{F\left(\omega^{\prime} \alpha\right)} X-\mathbb{1}_{F\left(\omega^{\prime} \alpha\right)} \eta \mid \omega^{\prime}\right)=0=\phi\left(\mathbb{1}_{F\left(\omega^{\prime} \alpha\right)} X \mid \omega^{\prime}\right) .
$$

Generally speaking, if we have $X \leq Y$ and $\phi(X)=\phi(Y)$ where $\phi$ is a strongly relevant coherent acceptability measure, then it follows that $X=Y$. Indeed, from superadditivity we know that $\phi(Y)=\phi((Y-X)+X) \geq \phi(Y-X)+\phi(X)$ so that from $Y \geq X$ and $\phi(Y)=\phi(X)$ we obtain $\phi(Y-X)=0$. Again because $Y-X \geq 0$, strong relevance now implies that $Y=X$. Applying this general rule to the situation in (A.5), we conclude that $\mathbb{1}_{F\left(\omega^{\prime} \alpha\right)} \eta=0$ so that $\eta=0$, which is what we needed to prove.

\section{Proof of Theorem 5.1}

Tower Law $\Rightarrow$ Sequential Consistency.

As shown in Roorda et al. (2004, Prop. 2.6), dynamic consistency is equivalent to stepwise monotonicity (3.1), and this property clearly implies Condition 4 in Theorem 4.2.

\section{Tower Law $\Rightarrow$ Conditional Consistency.}

This implication follows directly from Theorem 7.1. An alternative direct proof can be given as follows, by verifying Condition 1 in Theorem 4.3 for an acceptability measure $\phi$ that satisfies the tower law property. From the rule (2.7) and Condition 1 in Theorem 4.1, it follows that

$$
\phi\left(\mathbb{1}_{F\left(\omega^{\prime} \alpha\right)} X \mid \omega^{\prime}\right)=\phi\left(\mathcal{E}_{T\left(\omega^{\prime}\right)+1}^{\phi}\left(\mathbb{1}_{F\left(\omega^{\prime} \alpha\right)} X\right) \mid \omega^{\prime}\right)=\phi\left(\mathbb{1}_{F\left(\omega^{\prime} \alpha\right)} \phi\left(X \mid \omega^{\prime} \alpha\right) \mid \omega^{\prime}\right) .
$$

Write $Y:=\mathbb{1}_{F\left(\omega^{\prime} \alpha\right)} \phi\left(X \mid \omega^{\prime} \alpha\right)$. Condition 1 in Theorem 4.3 is proved if we show that $\phi\left(X \mid \omega^{\prime} \alpha\right) \geq 0$ if and only if $\phi\left(Y \mid \omega^{\prime}\right) \geq 0$. This is done as follows. If $\phi\left(X \mid \omega^{\prime} \alpha\right) \geq 0$, then $Y \geq 0$, so that by monotonicity of $\phi$ we have $\phi\left(Y \mid \omega^{\prime}\right) \geq 0$. On the other hand, if $\phi\left(X \mid \omega^{\prime} \alpha\right)<0$, then $Y \leq 0$ while $\mathbb{1}_{F\left(\omega^{\prime}\right)} Y \neq 0$, and consequently the relevance condition (3.2) implies that $\phi\left(Y \mid \omega^{\prime}\right)<0$.

Sequential Consistency $\Rightarrow$ Conditional Consistency (under strong relevance).

We verify Condition 1 in Theorem 4.3 from the single-step formulation of sequential consistency in Condition 2 of Theorem 4.2, assuming strong relevance.

Suppose first that $\phi\left(X \mid \omega^{\prime} \alpha\right) \geq 0$. Obviously, then $\phi\left(\mathbb{1}_{F\left(\omega^{\prime} \alpha^{\prime}\right)} X \mid \omega^{\prime} \alpha^{\prime}\right) \geq 0$ for all $\alpha^{\prime} \in A$, and from Condition 2 of Theorem 4.2 it follows that also $\phi\left(\mathbb{1}_{F\left(\omega^{\prime} \alpha\right)} X \mid \omega^{\prime}\right) \geq 0$.

Similarly, if $\phi\left(X \mid \omega^{\prime} \alpha\right)<0$, then $\phi\left(\mathbb{1}_{F\left(\omega^{\prime} \alpha^{\prime}\right)} X \mid \omega^{\prime} \alpha^{\prime}\right) \leq 0$ for all $\alpha^{\prime} \in A$, and it follows that $\phi\left(\mathbb{1}_{F\left(\omega^{\prime} \alpha\right)} X \mid \omega^{\prime}\right) \leq 0$. To arrive at Condition 1 in Theorem 4.3, we finally have to show that the last inequality is strict. Assume to the contrary to that $\phi\left(\mathbb{1}_{F\left(\omega^{\prime} \alpha\right)} X \mid \omega^{\prime}\right)=0$. From 
superadditivity of $\phi$, this assumption implies that

$$
\text { for all } \eta>0: \phi\left(\mathbb{1}_{F\left(\omega^{\prime} \alpha\right)}(X+\eta) \mid \omega^{\prime}\right) \geq \phi\left(\mathbb{1}_{F\left(\omega^{\prime} \alpha\right)} X \mid \omega^{\prime}\right)+\phi\left(\eta \mathbb{1}_{F\left(\omega^{\prime} \alpha\right)} \mid \omega^{\prime}\right)>0 \text {, }
$$

since, by assumption, the first term is zero, and the second term is strictly positive because of the strong relevance condition (3.3). However, then Condition 2 of Theorem 4.2 is violated, since for $0<\eta<\phi\left(\mathbb{1}_{F\left(\omega^{\prime} \alpha\right)} X \mid \omega^{\prime} \alpha\right)$, we have

$$
\max _{\alpha^{\prime} \in A} \phi\left(\mathbb{1}_{F\left(\omega^{\prime} \alpha\right)}(X+\eta) \mid \omega^{\prime} \alpha^{\prime}\right)=0<\phi\left(\mathbb{1}_{F\left(\omega^{\prime} \alpha\right)}(X+\eta) \mid \omega^{\prime}\right) .
$$

This completes the proof.

We end with a lemma that is needed in the proof of Thm. 4.2. The lemma shows that, when a global test set is replaced by its convex hull, the conditional test sets at the tree nodes are replaced by their convex hulls, so that the associated acceptability measure remains the same.

Lemma A.2 Let $\mathcal{P}$ be a global test set on $\Omega$. For all $\omega^{\prime} \in \Omega^{\prime}$, we have $(\operatorname{ch} \mathcal{P})\left(\omega^{\prime}\right)=$ $\operatorname{ch}\left(\mathcal{P}\left(\omega^{\prime}\right)\right)$.

Proof Take $\omega^{\prime} \in \Omega^{\prime}$. We first prove that $\operatorname{ch}\left(\mathcal{P}\left(\omega^{\prime}\right)\right) \subset(\operatorname{ch} \mathcal{P})\left(\omega^{\prime}\right)$. So, take $\mathbb{P} \in \operatorname{ch}\left(\mathcal{P}\left(\omega^{\prime}\right)\right)$; we need to show that there exists $\tilde{\mathbb{P}} \in \operatorname{ch}(\mathcal{P})$ such that $\mathbb{P}=\tilde{\mathbb{P}}\left(\cdot \mid \omega^{\prime}\right)$. By Carathéodory's theorem, the probability measure $\mathbb{P}$ can be written as a convex combination of finitely many measures in $\mathcal{P}\left(\omega^{\prime}\right)$ :

$$
\mathbb{P}=\sum_{i=1}^{N} \lambda_{i} \mathbb{P}_{i}\left(\cdot \mid \omega^{\prime}\right), \quad \mathbb{P}_{i} \in \mathcal{P}, \quad \mathbb{P}_{i}\left(\omega^{\prime}\right)>0, \quad \lambda_{i} \geq 0 \quad(i=1, \ldots, N), \quad \sum_{i} \lambda_{i}=1 .
$$

The numbers $\mu_{i}(i=1, \ldots, N)$ defined by

$$
\mu_{i}=\frac{\lambda_{i}\left(\mathbb{P}_{i}\left(\omega^{\prime}\right)\right)^{-1}}{\sum_{i=1}^{N} \lambda_{i}\left(\mathbb{P}_{i}\left(\omega^{\prime}\right)\right)^{-1}}
$$

satisfy $\mu_{i} \geq 0$ and $\sum_{i} \mu_{i}=1$. Note that

$$
\sum_{i} \mu_{i} \mathbb{P}_{i}\left(\omega^{\prime}\right)=\frac{\sum_{i} \lambda_{i}}{\sum_{i=1}^{N} \lambda_{i}\left(\mathbb{P}_{i}\left(\omega^{\prime}\right)\right)^{-1}}=\frac{1}{\sum_{i=1}^{N} \lambda_{i}\left(\mathbb{P}_{i}\left(\omega^{\prime}\right)\right)^{-1}}
$$

so that for all $i$

$$
\frac{\mu_{i}}{\sum_{i=1}^{N} \mu_{i} \mathbb{P}_{i}\left(\omega^{\prime}\right)}=\frac{\lambda_{i}}{\mathbb{P}_{i}\left(\omega^{\prime}\right)}
$$

Now, define $\tilde{\mathbb{P}}=\sum_{i=1}^{N} \mu_{i} \mathbb{P}_{i}$. For each $\omega \succeq \omega^{\prime}$, we have

$$
\tilde{\mathbb{P}}\left(\omega \mid \omega^{\prime}\right)=\frac{\tilde{\mathbb{P}}(\omega)}{\tilde{\mathbb{P}}\left(\omega^{\prime}\right)}=\frac{\sum_{i} \mu_{i} \mathbb{P}_{i}(\omega)}{\sum_{i} \mu_{i} \mathbb{P}_{i}\left(\omega^{\prime}\right)}=\sum_{i} \frac{\lambda_{i} \mathbb{P}_{i}(\omega)}{\mathbb{P}_{i}\left(\omega^{\prime}\right)}=\sum_{i} \lambda_{i} \mathbb{P}_{i}\left(\omega \mid \omega^{\prime}\right)=\mathbb{P}(\omega) .
$$

In other words, $\mathbb{P}=\sum \mu_{i} \mathbb{P}_{i}\left(\cdot \mid \omega^{\prime}\right) \in(\operatorname{ch} \mathcal{P})\left(\omega^{\prime}\right)$. 
The reverse inclusion is proved in a similar way. Take $\mathbb{P} \in \operatorname{ch} \mathcal{P}$; then there exist nonnegative numbers $\lambda_{1}, \ldots, \lambda_{N}$ and probability measures $\mathbb{P}_{1}, \ldots, \mathbb{P}_{N}$ in $\mathcal{P}$ such that $\sum_{i} \lambda_{i}=1$ and $\mathbb{P}=\sum_{i} \lambda_{i} \mathbb{P}_{i}$. Define numbers $\mu_{i}$ satisfying $\mu_{i} \geq 0$ and $\sum_{i} \mu_{i}=1$ by

$$
\mu_{i}=\frac{\lambda_{i} \mathbb{P}_{i}\left(\omega^{\prime}\right)}{\sum_{i=1}^{N} \lambda_{i} \mathbb{P}_{i}\left(\omega^{\prime}\right)} .
$$

We have, for $\omega \succeq \omega^{\prime}$,

$$
\sum_{i} \mu_{i} \mathbb{P}_{i}\left(\omega \mid \omega^{\prime}\right)=\sum_{i} \mu_{i} \frac{\mathbb{P}_{i}(\omega)}{\mathbb{P}_{i}\left(\omega^{\prime}\right)}=\frac{\sum_{i} \lambda_{i} \mathbb{P}_{i}(\omega)}{\sum_{i} \lambda_{i} \mathbb{P}_{i}\left(\omega^{\prime}\right)}=\frac{\mathbb{P}(\omega)}{\mathbb{P}\left(\omega^{\prime}\right)}=\mathbb{P}\left(\omega \mid \omega^{\prime}\right)
$$

Consequently, $\mathbb{P}\left(\cdot \mid \omega^{\prime}\right)=\sum_{i} \mu_{i} \mathbb{P}_{i}\left(\cdot \mid \omega^{\prime}\right) \in \operatorname{ch}\left(\mathcal{P}\left(\omega^{\prime}\right)\right)$.

\section{References}

Artzner, Ph., 2002. Conditional Value at Risk: Is it good in the multiperiod case? IIR Conference on Volatility and Risk, London, February 18-19.

Artzner, Ph., F. Delbaen, J.-M. Eber, and D. Heath, 1999. Coherent measures of risk. Mathematical Finance, 9, 203-228.

Artzner, Ph., F. Delbaen, J.-M. Eber, D. Heath, and H. Ku, 2004. Coherent multiperiod risk adjusted values and Bellman's principle. Manuscript, Institut de Recherche Mathématique Avancée, Strasbourg.

Carr, P., H. Geman, and D. B. Madan, 2001. Pricing and hedging in incomplete markets. Journal of Financial Economics, 32, 131-167.

Černý, A., and S. Hodges, 2002. The theory of good-deal pricing in financial markets. In: H. Geman, D. Madan, S.R. Pliska, T. Vorst (eds.), Mathematical Finance - Bachelier Congress 2000, Springer, Berlin, pp. 175-202.

Coquet, F., Y. Hu, J. Mémin, and S. Peng, 2002. Filtration-consistent nonlinear expectations and related $g$-expectations. Probability Theory and Related Fields, 123, 1-27.

Delbaen, F., 2002. Coherent risk measures on general probability spaces. In: K. Sandmann, P. J. Schönbucher (eds.), Advances in Finance and Stochastics. Essays in Honour of Dieter Sondermann, Springer, Berlin, pp.1-38.

Eichberger, J., S. Grant, and D. Kelsey, 2005. CEU preferences and dynamic consistency. Mathematical Social Sciences, 49, 143-151.

Epstein, L.G., and M. Schneider, 2003. Recursive multiple-priors. Journal of Economic Theory, 113, 1-31. 
Frittelli, M. and E. Rosazza Gianin, 2002. Putting order in risk measures. Journal of Banking and Finance, 26, 1473-1486.

Frittelli, M. and E. Rosazza Gianin, 2004. Dynamic convex risk measures. In: G. Szegö (ed.), Risk Measures for the 21st Century, Wiley, New York.

Frittelli, M. and G. Scandolo, 2004. Risk measures and capital requirements for processes. Manuscript, Università degli Studi di Firenze.

Föllmer, H., and A. Schied, 2002. Convex measures of risk and trading constraints. Finance and Stochastics, 6, 429-447.

Föllmer, H., and A. Schied, 2004. Stochastic Finance. An introduction in discrete time (2nd ed.). De Gruyter Studies in Mathematics, Vol. 27, de Gruyter, Berlin / New York.

Jaschke, S., and U. Küchler, 2001. Coherent risk measures and good-deal bounds. Finance and Stochastics, 5, 181-200.

Kaas, R., M. Goovaerts, J. Dhaene, and M. Denuit, 2003. Modern Actuarial Risk Theory, revised edition. Kluwer, Dordrecht.

Landsman, Z., and M. Sherris, 2001. Risk measures and insurance premium principles. Insurance: Mathematics and Economics, 29, 103-115.

Peng, S., 1997. BSDE and related g-expectation. In: N. El Karoui and L. Mazliak (eds.), Backward Stochastic Differential Equations, Pitman Research Notes in Mathematics, vol. 364, Longman, Harlow, 1997, 141-159.

Peng, S., 2004. Filtration consistent nonlinear expectations and evaluations. 3rd Workshop on Markov Processes and Related Topics, Beijing, August 10-14.

Riedel, F., 2004. Dynamic coherent risk measures. Stochastic Processes and their Applications, 112, 185-200.

Rockafellar, R. T., 1970. Convex Analysis. Princeton University Press, Princeton, NJ.

Rockafellar, R. T. and S. Uryasev, 2002. Conditional value-at-risk for general loss distributions. Journal of Banking and Finance, 26, 1443-1471.

Roorda, B., J. M. Schumacher, and J. C. Engwerda, 2004. Coherent acceptability measures in multiperiod models. To appear in Mathematical Finance.

Scandolo, G., 2003. Risk Measures in a Dynamic Setting. PhD thesis, Università degli Studi di Milano.

Staum, J., 2004. Fundamental theorems of asset pricing for good deal bounds. Mathematical Finance, 14-2, 141-161. 
Wang, S. S., V. R. Young, and H. H. Panjer, 1997. Axiomatic characterization of insurance prices. Insurance: Mathematics and Economics, 21, 173-183.

Wang, T., 2003. Conditional preferences and updating. Journal of Economic Theory, 108, $286-321$.

Weber, S., 2004. Distribution-invariant risk measures, information, and dynamic consistency. Manuscript, Humboldt-Universität zu Berlin.

Wu, G., 1999. Anxiety and decision making with delayed resolution of uncertainty. Theory and Decision, 46, 159-198. 